\title{
The 4-CB algebra and solvable lattice models
}

\author{
Vladimir Belavin, ${ }^{a, b, c}$ Doron Gepner, ${ }^{f}$ Jian-Rong $\mathbf{L i}^{d}$ and Ran Tessler ${ }^{e}$ \\ ${ }^{a}$ Physics Department, Ariel University, \\ Ariel 40700, territories administered by Israel \\ ${ }^{b}$ I.E. Tamm Department of Theoretical Physics, P.N. Lebedev Physical Institute, \\ Leninsky av. 53, 11991 Moscow, Russia \\ ${ }^{c}$ Department of Quantum Physics, Institute for Information Transmission Problems, \\ Bolshoy Karetny per. 19, 127994 Moscow, Russia \\ ${ }^{d}$ Institute of Mathematics and Scientific Computing, University of Graz, \\ Graz 8010, Austria \\ ${ }^{e}$ Department of Mathematics, Weizmann Institute, \\ Rehovot 76100, Israel \\ ${ }^{f}$ Department of Particle Physics and Astrophysics, Weizmann Institute, \\ Rehovot 76100, Israel \\ E-mail: vlbelavin@gmail.com, doron.gepner@weizmann.ac.il, \\ lijr07@gmail.com, ran.tessler@weizmann.ac.il
}

ABSTRACT: We study the algebras underlying solvable lattice models of the type fusion interaction round the face (IRF). We propose that the algebras are universal, depending only on the number of blocks, which is the degree of polynomial equation obeyed by the Boltzmann weights. Using the Yang-Baxter equation and the ansatz for the Baxterization of the models, we show that the three blocks models obey a version of Birman-MurakamiWenzl (BMW) algebra. For four blocks, we conjecture that the algebra, which is termed 4-CB (Conformal Braiding) algebra, is the BMW algebra with a different skein relation, along with one additional relation, and we provide evidence for this conjecture. We connect these algebras to knot theory by conjecturing new link invariants. The link invariants, in the case of four blocks, depend on three arbitrary parameters. We check our result for $G_{2}$ model with the seven dimensional representation and for $\mathrm{SU}(2)$ with the isospin $3 / 2$ representation, which are both four blocks theories.

KEYwords: Conformal Field Theory, Lattice Integrable Models, Integrable Field Theories

ArXiv EPRINT: 1909.02472 


\section{Contents}

1 Introduction $\quad 1$

1.1 Solvable IRF models 2

1.1.1 Interaction-round-the-face (IRF) models 2

1.1.2 Solvability, Yang-Baxter equation (YBE) and braiding 3

1.2 RCFTs and fusion IRF models 5

1.2.1 Braiding 6

$\begin{array}{ll}\text { 1.2.2 An ansatz for Baxterization } & 6\end{array}$

$1.3 n$ Conformal Braiding (CB) algebras 8

1.4 The main results 8

$2 n=3$ case $\quad 9$

3 The 4-CB algebra $r$

$3.1 n=4$ YBE and relations (3.11)-(3.21) 14

4 Three parameter link invariant $\quad 18$

$\begin{array}{lll}5 & G_{2} \text { IRF model } & 19\end{array}$

5.1 Kuperberg's $G_{2}$ link invariant 20

$\begin{array}{lll}6 & H \text { and } K \text { relations } & 21\end{array}$

$7 \quad H$ and $K$ relations for the general four blocks models 25

8 Conclusions 31

A Weights of the $G_{2}$ model

\section{Introduction}

Solvable lattice models are important as exact models of statistical physics, conformal field theory and phase transitions. For a review see [1]. These models are also connected to domains of mathematics, being of importance in subjects such as Rogers Ramanujan identities, knot theory, quantum algebra and probability theory.

Of particular interest, both in mathematics and physics, is the underlying algebraic structure of solvable lattice models. Our general idea is that the algebra depends only on the number of blocks, which is the degree of the polynomial equation obeyed by the Boltzmann weights, and is general to all the models, independently of the details of the model. This paper is directed towards proving this pivotal assertion. 
We started investigating this structure in the papers [2,3], based on the initial results of the work [4]. For two blocks, it is well known to be described by Templerley-Lieb algebra [5], or equivalently Hecke algebra. We study here the three and four blocks cases. We base our investigation on an ansatz for the Baxterisation put forward in ref. [4]. Using this ansatz and the Yang-Baxter equation (YBE), we show that the three blocks case obeys a weak version of the Birman-Murakami-Wenzl algebra (BMW) [6, 7]. This is discussed in section 2.

For the four blocks case, we find using the ansatz, an algebra which we call 4-CB (Conformal Braiding). This algebra includes the BMW algebra, with a different skein relation, along with one additional relation. This is described in section 3 . The connection of this algebraic structure to knot theory is described in section 4 .

We check the ansatz for $G_{2}$ theory, which is a four blocks theory, in section 5 . We already checked it for $\mathrm{SU}(2)$ with the field of isposin $3 / 2$ in ref. [3]. We find that it holds in both cases. We connect the algebraic structure with the tangle algebra of Kuperberg and Kalfagianni [8, 9]. We find that Kalfagianni's relations hold for any four blocks theory, assuming the ansatz, YBE and BMW. This is treated in sections 6-7.

The inversion relation method to compute the free energy, that we use, was first developed in ref. [10]. It was further elucidated by Baxter [11]. The Baxterization method that we propose here, was first suggested by Jones for the two block case [12].

Our approach to knot invariants is quite similar to that of [13] and references therein. These authors construct a Markov trace for any solvable lattice model and prove that it obeys the Markov properties, thus defining a link invariant for any solvable lattice model. An equivalent approach is that of Reshetikhin and Turaev [14]. Thes authors define a link invariant for any quantum group and any representation. Presumably, the two approaches give the same link invariants.

We define link invariants based on the Baxterization ansatz that we proposed. We assume that the conjecture that we have a Markov trace, described in section 4, is correct. Then our link invaraint differs from the above works in two ways. First, we give a tangle algebra that allows for the practical calculation of the invariants. Second, our link invariants depend on three arbitrary parameters, whereas the above works hold only for certain values of the parameters, which correspond to some lattice model or quantum group.

\subsection{Solvable IRF models}

\subsubsection{Interaction-round-the-face (IRF) models}

Let $I$ be a set, which is assumed to be finite for the purposes of this article. We assume that $I$ is endowed with two relations $\sim_{h}, \sim_{v}$. When $a \sim_{h} b\left(a \sim_{v} b\right)$ we say that $(a, b)$ are horizontally (vertically) admissible. In the special case $\sim_{h} \equiv \sim_{v}$, which will be mainly considered here, we write $\sim$ for $\sim_{h}, \sim_{v}$ and we say that $a, b$ are admissible whenever $a \sim b$. The third piece of data we require is a function, called the Boltzmann weight, of four elements of $a, b, c, d \in I$ and a complex parameter $u$, which is required to satisfy

$$
\omega\left(\begin{array}{ll}
a & b \\
c & d
\end{array} \mid u\right)=0,
$$


unless the admissibility condition

$$
a \sim_{h} b, a \sim_{v} c, c \sim_{h} d, b \sim_{v} d
$$

is met. The parameter $u$ is called the spectral parameter.

An interaction-round-the-face (IRF) lattice model is defined on a two dimensional square lattice, or its finite approximation via a $M \times M$ box with periodic boundary conditions (we will not make use in the periodicity in the algebraic treatment below, and will keep it only for the combinatorial motivation). We denote the underlying graph in both cases by $T$. A configuration is an assignment of an element of $I$ to each vertex of $T$. The partition function of the model is defined to be

$$
Z=Z(u)=Z_{T}(u):=\sum_{\text {configurations faces }} \prod_{c} \omega\left(\begin{array}{ll}
a & b \\
c & d
\end{array} \mid u\right),
$$

The state space of the theory is $\left(\mathbf{C}^{I}\right)^{\otimes N}$, and we denote states using the ket notation $\left|a_{1} \ldots a_{N}\right\rangle$. Dual states are denoted using the bra notation, $\left\langle a_{1} \ldots a_{N}\right|$. A state is $a d-$ missible if for all $i, a_{i} \sim a_{i \pm 1}$ (we assume here $\sim_{h}=\sim_{v}$, otherwise there are analogous requirements, but which depend on the parity of $i$ ). Denote by $V_{a d m}$ the space spanned by the admissible states.

\subsubsection{Solvability, Yang-Baxter equation (YBE) and braiding}

We define the $i^{\text {th }}$ face transfer matrix $X_{i}(u)$ by

$$
\left\langle a_{1}, a_{2}, \ldots, a_{N}\left|X_{i}(u)\right| a_{1}^{\prime}, a_{2}^{\prime}, \ldots, a_{N}^{\prime}\right\rangle=\left[\prod_{j \neq i} \delta_{a_{j}, a_{j}^{\prime}}\right] \omega\left(\begin{array}{cc}
a_{i-1} & a_{i} \\
a_{i}^{\prime} & a_{i+1}
\end{array} \mid u\right) .
$$

For any $u, v$, we have

$$
X_{i}(u) X_{j}(v)=X_{j}(v) X_{i}(u), \quad j \neq i \pm 1 .
$$

The Yang-Baxter equation (YBE) is the relation

$$
X_{i}(u) X_{i+1}(u+v) X_{i}(v)=X_{i+1}(v) X_{i}(u+v) X_{i+1}(u) .
$$

An equivalent formulation of this equation, in terms of the Boltzmann weights, is

$$
\begin{aligned}
\sum_{c} \omega\left(\begin{array}{ll}
g & c \\
a & b
\end{array} \mid u\right) \omega\left(\begin{array}{ll}
c & e \\
b & d
\end{array} \mid u+v\right) \omega\left(\begin{array}{ll}
g & f \\
c & e
\end{array} \mid v\right)= \\
=\sum_{c} \omega\left(\begin{array}{ll}
a & c \\
b & d
\end{array} \mid v\right) \omega\left(\begin{array}{ll}
g & f \\
a & c
\end{array} \mid u+v\right) \omega\left(\begin{array}{ll}
f & e \\
c & d
\end{array} \mid u\right) .
\end{aligned}
$$

If the YBE is satisfied then transfer matrices for different spectral parameters commute. 
Remark 1. Although the point of view presented in this paper is algebraic, we make a small digression concerning the combinatorial picture. A front in a $M \times M$ box $T$ with periodic boundary conditions (or equivalently a $M \times M$ torus) is a chain of $N=2 M-2$ vertices $v_{1}, \ldots, v_{N}$ such that $v_{i}$ is a neighbor of $v_{i+1}$, where addition is modulo $N$, and such that the projections of $v_{1}, \ldots, v_{N}$ on the diagonal $x=-y$ are increasing, when the diagonal is oriented from NW to SE. An example of a front is the standard front, which is an arbitrary shift of the vertices

$$
(0, M),(0, M-1)(1, M-1),(1, M-2), \ldots(M, 0)=(0, M)
$$

by a lattice vector. A state can be thought of as an association of an element of $I$ to each vertex of the front. The state is admissible if neighboring elements are. Since all fronts are of the same size, there are isomorphisms between their state spaces.

The $i^{\text {th }}$ face transfer matrix should be thought as promoting the front from $\left(v_{1}, \ldots, v_{i-1}, v_{i}, v_{i+1}, \ldots, v_{N}\right)$ to $\left(v_{1}, \ldots, v_{i-1}, v_{i}, v_{i+1}, \ldots, v_{N}\right)$ by adding a square whose four vertices are

$$
v_{i}, \quad v_{i-1}=v_{i}+(1,0), \quad v_{i+1}=v_{i}+(0,1), \quad v_{i}, \quad v_{i}^{\prime}=v_{i}+(1,1) .
$$

We see that the face transfer matrices are operators between different, although isomorphic, state spaces.

Starting from a standard front, applying the transfer matrix

$$
X=X(u)=X_{1}(u) \cdot X_{3}(u) \cdot X_{5}(u) \cdots
$$

amounts to pushing the front by $(1,0)$ and cyclically shifting indices by 1 . Thus, roughly speaking

$$
Z_{T}(u)=\operatorname{Tr}\left(X^{M}\right)
$$

This means that if $\mu_{1}(u), \ldots, \mu_{r}(u)$ are the eigenvalues of $X$, counted with multiplicities, the partition function equals

$$
Z(u)=\sum_{i=1}^{r} \mu_{i}(u)^{M} .
$$

If one can understand the eigenvalues of the transfer matrix good enough, the model is usually solvable, meaning that its partition function can be calculated. It follows from direct calculation that when the Yang-Baxter equation (1.5) and the commutation equation (1.4) hold, the matrices $X(u)$ for different $u$ commute. This means that they have common eigenspaces. In many occasions the combinatorics or physics of the IRF model give rise to additional constrains on the transfer matrices, which in turn give rise to functional equations satisfies by the different $\mu_{i}(u)$. Sometimes these constraints are strong enough to determine the eigenvalues.

One such constraint may be an inversion relation which connects $X_{i}(u)$ and $X_{i}(-u)$. We will consider such an inversion relation below. More details about the transfer matrix method in statistical mechanics models, as well as different inversion relations can be found in ref. [1]. 
Suppose that the UV limit of the face transfer matrices

$$
X_{i}=\lim _{u \rightarrow i \infty} g(u) X_{i}(u),
$$

exists and is finite and non zero, where $g(u)$ is some function. Then the matrix coefficients of $X_{i}$ automatically satisfy the admissibility conditions equation (1.1). In addition, one can also take the limits of equations (1.4), (1.5) to obtain

$$
\begin{aligned}
X_{i} X_{j} & =X_{j} X_{i}, & j \neq i \pm 1 . \\
X_{i} X_{i+1} X_{i} & =X_{i+1} X_{i} X_{i+1} . &
\end{aligned}
$$

These equations imply that $X_{1}, \ldots, X_{N}$ form a representation of the braid group.

\subsection{RCFTs and fusion IRF models}

In ref. [4] a conjectural recipe for obtaining solvable IRF models from a rational conformal field theory was described. We review it here, but refer to ref. [4] for more details. We begin by providing a very partial definition of conformal field theories, and recalling the most basic properties we need for the discussion below. We refer the reader to ref. [15] for a complete definition and extensive analysis of conformal field theories.

A conformal field theory (CFT) $\mathcal{O}$ is a collection of primary fields, labelled by a set $I$, together with a fusion product, which is a commutative and associative product defined via the fusion structure constants $f_{a b}^{c}(a, b, c$ are elements of $I)$

$$
a \times b=f_{a b}^{c} c .
$$

Each primary field has a conformal dimension which is a non negative rational number which specifies the behaviour of the field under conformal symmetries. We identify elements of $I$ with the corresponding primary fields. $\mathcal{O}$ is a rational conformal field theory (RCFT) if $I$ is finite.

Given a RCFT $\mathcal{O}$ and two fields $h, v$ one can use the fusion product to write admissibility conditions $\sim_{h}, \sim_{v}$ as follows:

$$
a \sim_{h} b \Leftrightarrow f_{a h}^{b}>0, \quad a \sim_{h} b \Leftrightarrow f_{a v}^{b}>0 .
$$

An IRF model with a set of states $I$ and the admissibility conditions above is called a fusion IRF model. Such a model is completely specified by its Boltzmann weights $\omega\left(\begin{array}{ll}a & b \\ c & d\end{array} \mid u\right)$ which vanish unless

$$
f_{a h}^{b}>0, f_{c h}^{d}>0, \quad f_{a v}^{c}>0, \text { and } f_{b v}^{d}>0
$$

A fusion IRF model is called a $n$ conformal braiding (CB) IRF if the fusion product of the primary fields $h$ and $v$ is a sum of $n$ primary fields

$$
[h] \times[v]=\sum_{i=0}^{n-1} \psi_{i}
$$




\subsubsection{Braiding}

We are interested in constructing solvable fusion IRF models, meaning models for which the Bolzmann weights satisfy (1.6). As we saw above, for such a model, if one can define the UV limit, a representation of the braid group appears, and it is a representation in which the matrix components satisfy the admissibility conditions (1.10). To a conformal field theory there are associated braiding matrices

$$
C_{c, d}\left[\begin{array}{ll}
h & v \\
a & b
\end{array}\right] .
$$

The matrix components of these matrices vanish unless the admissibility conditions (1.10) holds. In addition, these matrices satisfy the hexagon relation, and when $h=v$, this relation reduces to the braiding relations (1.8), (1.9).

Moreover, it can be shown that the matrix $C$ whose components are given by (1.12) for fixed $h, v$ satisfies the characteristic equation

$$
\prod_{i=0}^{n-1}\left(C-\lambda_{i}\right)=0,
$$

where $n$ is the number of blocks, and $\lambda_{i}$ are given by

$$
\lambda_{i}=\epsilon_{i} e^{i \pi\left(\Delta_{h}+\Delta_{v}-\Delta_{i}\right)},
$$

here $\Delta_{i}, \Delta_{h}, \Delta_{v}$ are the conformal dimensions of $\psi_{i}, h, v$ respectively, and $\epsilon_{i}= \pm 1$ according to whether the product is symmetric or anti-symmetric.

\subsubsection{An ansatz for Baxterization}

From now on we consider $h=\bar{h}=v$. The appearance of the natural braiding matrix suggests searching for a solvable fusion IRF model with this matrix as the UV limit. More precisely, we start with matrices $X_{i}, i=1, \ldots, N$, which are given by equation (1.3) with $\omega\left(\begin{array}{ll}a & b \\ c & d\end{array} \mid u\right)$ replaced by $C\left(\begin{array}{ll}a & b \\ c & d\end{array}\right):=C_{c, d}\left[\begin{array}{ll}h & h \\ a & b\end{array}\right]$.

These matrices satisfy the braiding equations (1.8), (1.9), the admissibility condition (1.10), and correspond to $n$ conformal blocks, meaning

$$
\prod_{p=0}^{n-1}\left(X_{i}-\lambda_{p}\right)=0,
$$

where $\lambda_{i}$ are given in equation (1.13). The goal is to construct matrices $X_{i}(u)$, which satisfy the admissibility relation (1.10), equations (1.4), (1.5), and that their UV limit is

$$
\lim _{u \rightarrow i \infty} g(u) X_{i}(u)=X_{i}
$$

for some function $g(u)$. The process of extending a representation $X_{i}, i=1, \ldots, N$ of the braid group, to a solution $X_{i}(u)$, of the Yang-Baxter equation (1.5) is called a Baxterization. 
The ansatz of ref. [4] is the following. Observe that

$$
X_{i}=\sum_{a=0}^{n-1} \lambda_{a} P_{i}^{a}
$$

where the projection $P_{i}^{a}$ to the $a^{\text {th }}$ eigenspace is given by

$$
P_{i}^{a}=\prod_{p \neq a}\left[\frac{X_{i}-\lambda_{p}}{\lambda_{a}-\lambda_{p}}\right]
$$

These projections satisfy the relations

$$
\sum_{a=0}^{n-1} P_{i}^{a}=1_{i}, \quad P_{i}^{a} P_{i}^{b}=\delta_{a, b} P_{i}^{b} .
$$

Remark 2. The operator $1_{i}$ is just the identity on the space of admissible states, and 0 on the space spanned by the complementary states. In the algebraic analysis we will conduct in the following sections, since we will restrict only to admissible states, and the face transfer matrices preserve $V_{a d m}$, we will be able to identify $1_{i}$ with the identity operator. The reason we still use the notation $1_{i}$ is that, as was explained in Remark 1 above, although we identify state spaces of different fronts with $V_{a d m}$, combinatorially it is more accurate to consider our operators are relating state spaces of different fronts. With this point of view $1_{i}$ has the meaning of a unit face transfer matrix, which promotes the front from $\left(v_{1}, \ldots, v_{i-1}, v_{i}, v_{i+1}, \ldots, v_{N}\right)$ to $\left(v_{1}, \ldots, v_{i-1}, v_{i}^{\prime}, v_{i+1}, \ldots, v_{N}\right)$ by adding a square whose four vertices are

$$
v_{i}, \quad v_{i-1}=v_{i}+(1,0), \quad v_{i+1}=v_{i}+(0,1), \quad v_{i}, \quad v_{i}^{\prime}=v_{i}+(1,1),
$$

and the field which is assigned to $v_{i}^{\prime}$ in the admissible case is the same field that is assigned to $v_{i}$.

We define the crossing parameters as

$$
\zeta_{i}=\pi\left(\Delta_{i+1}-\Delta_{i}\right) / 2, \quad \lambda=\zeta_{0} .
$$

The trigonometric ansatz for the Yang Baxter equations (1.5) is

$$
X_{i}(u)=\sum_{a=0}^{n-1} f_{a}(u) P_{i}^{a}
$$

where the functions $f_{a}(u)$ are given by

$$
f_{a}(u)=\left[\prod_{r=1}^{a} \sin \left(\zeta_{r-1}-u\right)\right]\left[\prod_{r=a+1}^{n-1} \sin \left(\zeta_{r-1}+u\right)\right] /\left[\prod_{r=1}^{n-1} \sin \left(\zeta_{r-1}\right)\right] .
$$

With this ansatz the following inversion relation or unitarity is straight forward

$$
X_{i}(u) X_{i}(-u)=\rho(u) \rho(-u) 1_{i}
$$


where the function $\rho$ is defined by

$$
\rho(u)=\prod_{r=1}^{n-1} \frac{\sin \left(\zeta_{r-1}-u\right)}{\sin \left(\zeta_{r-1}\right)} .
$$

It is also conjectured in ref. [4] that the Boltzmann weights obey crossing symmetry

$$
\omega\left(\begin{array}{ll}
a & b \\
c & d
\end{array} \mid \lambda-u\right)=\left[\frac{G_{b} G_{c}}{G_{a} G_{d}}\right]^{1 / 2} \omega\left(\begin{array}{ll}
c & a \\
d & b
\end{array} \mid u\right),
$$

where $G_{a}$ is some factor and $\lambda=\zeta_{0}$ is the crossing parameter.

The IRF model given by this ansatz is called the $(\mathcal{O}, h, v)$ fusion IRF model.

\section{3 n Conformal Braiding (CB) algebras}

The algebras formed by the operators $X_{i}(u), i=1, \ldots, N$, in the $n$ blocks case are collectively called $n \mathrm{CB}$ algebras. We would like to understand the structure of these algebras, what are the relations between generators, and whether there are interesting subalgebras or quotients.

The simplest non trivial case is the $n=2$ case. In this case $X_{i}$ satisfy a quadratic relation, and it is shown in ref. [4] section 7, that the algebra formed by the $X_{i}$ is a $A_{N+1}-$ Hecke algebra. In this case it is also proven that the ansatz provides a solution to the YBE (1.5) and the commutation (1.4).

An example of an interesting subalgebra is an embedding of the Temperley-Lieb algebra: we define the operator,

$$
E_{i}=X_{i}(\lambda)
$$

where $\lambda$ is the crossing parameter of (1.19). Assuming the crossing symmetry (1.24) holds, it follows that

$$
E\left(\begin{array}{ll}
a & b \\
c & d
\end{array}\right)=\left(\frac{G_{b} G_{c}}{G_{a} G_{d}}\right)^{1 / 2} \delta_{a, d}
$$

where we denoted $E_{i}$ above with its explicit indices. From this equation, it follows, that $E_{i}$ obeys the Temperley-Lieb algebra (for any $n$ ),

$$
E_{i} E_{i \pm 1} E_{i}=E_{i}, \quad E_{i}^{2}=b E_{i}, \quad E_{i} E_{j}=E_{j} E_{i}, \quad \text { if }|i-j|>1,
$$

where

$$
b=\prod_{r=0}^{n-2} \frac{\sin \left(\lambda+\zeta_{r}\right)}{\sin \left(\zeta_{r}\right)}
$$

\subsection{The main results}

In this paper we analyze $n \mathrm{CB}$ algebras for $n=3,4$ (and $h=v=\bar{h}$ ).

In the $n=3$ case we prove that the operators $X_{i}(u)$ constructed by the ansatz satisfy the required relations (1.4), (1.5) and (1.24) if an only if the generators $1, E_{i}, G_{i}, G_{i}^{-1}, i=$ $1, \ldots, N$ where $G_{i}^{ \pm 1}$ are proportional to $X_{i}, X_{i}^{t}$, form an algebra to which we call the weak Birman-Murakami-Wenzl (BMW) algebra. This algebra which is defined below, has 
the property that a simple quotient of it gives the well known BMW algebra $[6,7]$. We conjecture, and have verified in many examples, that the above generators satisfy the BMW algebra itself. We conjecture that most of the BMW algebra relations hold for all $n$.

In the $n=4$ case, assuming this conjecture regarding the BMW relations for general $n$, we describe an algebra which is a generalization of the BMW algebra over the same set of generators, and which is equivalent to YBE.

We then consider two explicit special cases with $n=4$ blocks. The first is the $G_{2}$ model and the second is $\mathrm{SU}(2) 3 \times 3$ model. For the first model we show that by adding two new types of generators $H_{i}, K_{i}, i=1, \ldots, N$ the algebra formed by $1, E_{i}, G_{i}, G_{i}^{-1}, H_{i}, K_{i}, i=$ $1, \ldots, N$ is the Kalfagianni-Kuperberg algebra (defined in [9], following the work of ref. [8]). We then show that by defining $H_{i}, K_{i}$ in an analogous way, very similar relations hold in the case of $\mathrm{SU}(2) 3 \times 3$ model. The algebra we find there is new, as far as we know. We extend the definition of the new generators $H_{i}, K_{i}$ to any $n=4$ fusion IRF model. In sections 6,7 we show that the relations we find also extend in a similar manner to the general $n=4$ theories.

\section{$2 n=3$ case}

Consider the case of $n=3$. Auppose the crossing relation

$$
\omega\left(\begin{array}{ll}
a & b \\
c & d
\end{array} \mid \zeta_{0}-u\right)=\left(\frac{G_{b} G_{c}}{G_{a} G_{d}}\right)^{\frac{1}{2}} \omega\left(\begin{array}{ll}
c & a \\
d & b
\end{array} \mid u\right),
$$

where $G_{a}$ is the multiplier.

Set $s_{0}=e^{i \zeta_{0}}, s_{1}=e^{i \zeta_{1}}$.

Recall equation (1.25) and the ansatz. We have

$$
E_{i}=X_{i}\left(\zeta_{0}\right)=\frac{\sin \left(2 \zeta_{0}\right) \sin \left(\zeta_{0}+\zeta_{1}\right)}{\sin \left(\zeta_{0}\right) \sin \left(\zeta_{1}\right)} P_{i}^{0}=\frac{\left(s_{0}^{2} s_{1}^{2}-1\right)\left(s_{0}^{2}+1\right)}{\left(s_{1}^{2}-1\right) s_{0}^{2}} P_{i}^{0} .
$$

Put

$$
\begin{aligned}
G_{i}=4 \sin \left(\zeta_{0}\right) \sin \left(\zeta_{1}\right) e^{-i \zeta_{0}} X_{i} & =-e^{-2 i \zeta_{0}-i \zeta_{1}} P_{i}^{0}+e^{-i \zeta_{1}} P_{i}^{1}-e^{i \zeta_{1}} P_{i}^{2} \\
& =-s_{0}^{-2} s_{1}^{-1} P_{i}^{0}+s_{1}^{-1} P_{i}^{1}-s_{1} P_{i}^{2}
\end{aligned}
$$

Then

$$
\begin{aligned}
P_{i}^{0} & =\frac{\left(s_{1}^{2}-1\right) s_{0}^{2}}{\left(s_{0}^{2} s_{1}^{2}-1\right)\left(s_{0}^{2}+1\right)} E_{i} \\
P_{i}^{1} & =\frac{s_{1}^{2}-1}{\left(s_{0}^{2}+1\right)\left(s_{1}^{2}+1\right) s_{0}^{2}} E_{i}+\frac{s_{1}^{2}}{s_{1}^{2}+1} G_{i}^{2}+\frac{s_{1}^{3}}{s_{1}^{2}+1} G_{i} \\
P_{i}^{2} & =-\frac{\left(s_{1}^{2}-1\right)}{\left(s_{0}^{2} s_{1}^{2}-1\right)\left(s_{1}^{2}+1\right) s_{0}^{2} s_{1}^{2}} E_{i}-\frac{1}{\left(s_{1}^{3}+s_{1}\right)} G_{i}+\frac{1}{s_{1}^{2}+1} G_{i}^{2}
\end{aligned}
$$

and $G_{i} E_{i}=E_{i} G_{i}=l^{-1} E_{i}$, where $l=-s_{0}^{2} s_{1}$.

Using $P_{i}^{0}+P_{i}^{1}+P_{i}^{2}=1$, we obtain

$$
G_{i}^{2}=-\frac{\left(s_{1}^{2}-1\right)}{s_{0}^{2} s_{1}^{2}} E_{i}+1+\left(\frac{1}{s_{1}}-s_{1}\right) G_{i}
$$


Denote $m=-2 i \sin \left(\zeta_{1}\right)=s_{1}^{-1}-s_{1}$. Then

$$
G_{i}=-\frac{m}{l} E_{i} G_{i}^{-1}+G_{i}^{-1}+m
$$

This implies the skein relation

$$
m\left(E_{i}-1\right)=G_{i}^{-1}-G_{i}
$$

Using the expression for $G_{i}^{2}$, we have

$$
\begin{aligned}
P_{i}^{0} & =\frac{\left(s_{1}^{2}-1\right) s_{0}^{2}}{\left(s_{0}^{2} s_{1}^{2}-1\right)\left(s_{0}^{2}+1\right)} E_{i}, \\
P_{i}^{1} & =-\frac{\left(s_{1}^{2}-1\right)}{\left(s_{0}^{2}+1\right)\left(s_{1}^{2}+1\right)} E_{i}+\frac{s_{1}^{2}}{s_{1}^{2}+1}+\frac{s_{1}}{s_{1}^{2}+1} G_{i} \\
P_{i}^{2} & =-\frac{\left(s_{1}^{2}-1\right)}{\left(s_{0}^{2} s_{1}^{2}-1\right)\left(s_{1}^{2}+1\right)} E_{i}-\frac{s_{1}}{\left(s_{1}^{2}+1\right)} G_{i}+\frac{1}{s_{1}^{2}+1} .
\end{aligned}
$$

Therefore

$$
\begin{aligned}
X_{i}(u) & =\frac{1}{\left(s_{0}^{2}-1\right)\left(s_{1}^{2}-1\right)}\left(q_{1}+q_{2} e^{2 i u}+q_{3} e^{-2 i u}\right), \\
q_{1} & =\left(s_{1}^{2}-1\right) s_{0}^{2}+\left(1-s_{1}^{2}\right) E_{i}+\left(s_{0}^{2}+1\right) s_{1} G_{i}, \\
q_{2} & =\left(1-s_{1}^{2}\right)+\left(s_{1}^{2}-1\right) E_{i}-s_{1} G_{i}, \\
q_{3} & =-s_{0}^{2} s_{1} G_{i} .
\end{aligned}
$$

By equations (1.26-1.27), $E_{i} E_{j} E_{i}=E_{i}$, when $|i-j|=1$, and $E_{i}^{2}=b E_{i}$, where $b=\frac{\sin \left(2 \zeta_{0}\right) \sin \left(\zeta_{0}+\zeta_{1}\right)}{\sin \left(\zeta_{0}\right) \sin \left(\zeta_{1}\right)}=\frac{\left(s_{0}^{2} s_{1}^{2}-1\right)\left(s_{0}^{2}+1\right)}{\left(s_{1}^{2}-1\right) s_{0}{ }^{2}}$.

The skein relation implies that for $|i-j|=1$,

$$
\begin{aligned}
\left(E_{i}-1\right) E_{j} G_{i} & =\frac{1}{m}\left(G_{i}^{-1}-G_{i}\right) E_{j} G_{i} \\
G_{i} E_{j}\left(E_{i}-1\right) & =\frac{1}{m} G_{i} E_{j}\left(G_{i}^{-1}-G_{i}\right),
\end{aligned}
$$

and

$$
G_{i}^{-1} E_{j} G_{i}=G_{i}^{-1}\left(1+\frac{1}{m}\left(G_{j}^{-1}-G_{j}\right)\right) G_{i}, \quad G_{j} E_{i} G_{j}^{-1}=G_{j}\left(1+\frac{1}{m}\left(G_{i}^{-1}-G_{i}\right)\right) G_{j}^{-1} .
$$

Therefore

$$
\begin{aligned}
& E_{i} E_{j} G_{i}=E_{j} G_{i}-\frac{1}{m} G_{i} E_{j} G_{i}+\frac{1}{m} G_{i}^{-1} E_{j} G_{i}, \\
& G_{i} E_{j} E_{i}=G_{i} E_{j}+\frac{1}{m} G_{i} E_{j} G_{i}^{-1}-\frac{1}{m} G_{i} E_{j} G_{i} .
\end{aligned}
$$

and $G_{i}^{-1} E_{j} G_{i}=G_{j} E_{i} G_{j}^{-1}$. 
Using $X_{i}(u) X_{i+1}(u+v) X_{i}(v)=X_{i+1}(v) X_{i}(u+v) X_{i+1}(u)$ and the above relations, we obtain 19 equations. The only two independent equations are the following equations.

$$
\begin{aligned}
& \left(s_{1}^{2}-1\right)^{2}\left(s_{0}^{2} s_{1}^{2}-s_{1}^{2}+1\right) E_{i}-\left(s_{1}^{2}-1\right)^{2}\left(s_{0}^{2} s_{1}^{2}-s_{1}{ }^{2}+1\right) E_{i+1} \\
& +\left(s_{1}{ }^{2}-1\right)^{2} s_{1} E_{i+1} G_{i}+\left(s_{1}{ }^{4}-s_{1}{ }^{2}\right) G_{i} E_{i+1} G_{i}+\left(s_{1}{ }^{2}-1\right)^{2} s_{1} E_{i} G_{i+1} E_{i} \\
& +\left(s_{1}^{2}-1\right)^{2} s_{1} G_{i} E_{i+1}-\left(s_{1}^{2}-1\right)^{2} s_{1} E_{i} G_{i+1}+\left(s_{1}{ }^{2}-s_{1}^{4}\right) G_{i+1} E_{i} G_{i+1} \\
& -\left(s_{1}{ }^{2}-1\right)^{2} s_{1} E_{i+1} G_{i} E_{i+1}-\left(s_{1}{ }^{2}-1\right)^{2} s_{1} G_{i+1} E_{i}=0, \\
& -\left(s_{1}^{2}-1\right)^{2}\left(s_{0}^{4}+s_{0}^{2} s_{1}^{2}-s_{1}^{2}+1\right) E_{i}+\left(s_{1}^{2}-1\right)^{2}\left(s_{0}{ }^{4}+s_{0}{ }^{2} s_{1}{ }^{2}-s_{1}{ }^{2}+1\right) E_{i+1} \\
& -\left(s_{1}{ }^{2}-1\right)^{2}\left(s_{0}^{4}+1\right) s_{1} E_{i+1} G_{i}+\left(s_{1}{ }^{2}-1\right)^{2}\left(s_{0}^{4}+1\right) s_{1} G_{i+1} E_{i} \\
& -\left(s_{1}^{2}-1\right)\left(s_{0}^{4}+1\right) s_{1}^{2} G_{i} E_{i+1} G_{i}-\left(s_{0}^{2}+1\right)\left(s_{1}^{2}-1\right)^{2} s_{1} E_{i} G_{i+1} E_{i} \\
& -\left(s_{1}^{2}-1\right)^{2}\left(s_{0}^{4}+1\right) s_{1} G_{i} E_{i+1}+\left(s_{1}^{2}-1\right)^{2}\left(s_{0}^{4}+1\right) s_{1} E_{i} G_{i+1} \\
& +\left(s_{1}^{2}-1\right)\left(s_{0}^{4}+1\right) s_{1}^{2} G_{i+1} E_{i} G_{i+1}+\left(s_{0}^{2}+1\right)\left(s_{1}^{2}-1\right)^{2} s_{1} E_{i+1} G_{i} E_{i+1}=0 \text {. }
\end{aligned}
$$

These equations are equivalent to the following:

$$
s_{0}^{2} s_{1} E_{i}-s_{0}^{2} s_{1} E_{i+1}+E_{i} G_{i+1} E_{i}-E_{i+1} G_{i} E_{i+1}=0
$$

and

$$
\begin{gathered}
\left(s_{1}^{2}-1\right)^{2} E_{i}-\left(s_{1}{ }^{2}-1\right)^{2} E_{i+1}+\left(s_{1}-s_{1}^{3}\right) E_{i+1} G_{i}+\left(s_{1}{ }^{2}-1\right) s_{1} G_{i+1} E_{i} \\
-s_{1}{ }^{2} G_{i} E_{i+1} G_{i}+\left(s_{1}-s_{1}^{3}\right) G_{i} E_{i+1}+\left(s_{1}{ }^{2}-1\right) s_{1} E_{i} G_{i+1}+s_{1}{ }^{2} G_{i+1} E_{i} G_{i+1}=0 .
\end{gathered}
$$

Thus, assume the crossing relation (1.24), or even only its consequence eq. (1.26). We have proved that $X_{i}(u)$ satisfies the Yang-Baxter equation

$$
X_{i}(u) X_{i+1}(u+v) X_{i}(v)=X_{i+1}(v) X_{i}(u+v) X_{i}(u),
$$

if and only if $G_{i}, E_{i}$ satisfies the following relations:

$$
\begin{aligned}
G_{i} G_{j} & =G_{j} G_{i}, & & |i-j| \geq 2, \\
G_{i} G_{j} G_{i} & =G_{j} G_{i} G_{j}, & & |i-j|=1, \\
m\left(E_{i}-1\right) & =G_{i}^{-1}-G_{i}, & & \\
G_{i} E_{i} & =l^{-1} E_{i}, & & (2.18) \\
E_{i} G_{j} E_{i}-l E_{i}-E_{j} G_{i} E_{j}+l E_{j} & =0, & & |i-j|=1,
\end{aligned}
$$$$
m^{2}\left(E_{i}-E_{j}\right)+m\left(E_{j} G_{i}-G_{j} E_{i}+G_{i} E_{j}-E_{i} G_{j}\right)-G_{i} E_{j} G_{i}+G_{j} E_{i} G_{j}=0, \quad|i-j|=1,
$$

where $m=\frac{l-l^{-1}}{b-1}=s_{1}^{-1}-s_{1}, l=-s_{0}^{2} s_{1}$.

We call the above relations weak BMW relation and we call the algebra generated by $G_{i}, E_{i}$ subject to the weak BMW relation the weak BMW algebra.

In fact, for general $n$ blocks, using the ansatz, the Yang-Baxter equation implies that

$$
\begin{array}{rlrl}
G_{i} G_{j} & =G_{j} G_{i}, \quad|i-j| \geq 2, \\
G_{i} G_{j} G_{i} & =G_{j} G_{i} G_{j}, \quad|i-j|=1, \\
G_{i} E_{i} & =l^{-1} E_{i}, &
\end{array}
$$

for some $l$. 


\section{The 4-CB algebra}

We focus now on the four blocks case, $n=4$.

We find it convenient, for future use, to scale the braiding matrices as follows. We have

$$
X_{i}=\lim _{u \rightarrow i \infty} \exp (3 i u) X_{i}(u), \quad X_{i}^{t}=\lim _{u \rightarrow-i \infty} \exp (-3 i u) X_{i}(u) .
$$

We define the operators $G_{i}, G_{i}^{-1}$ and $E_{i}$ by,

$$
\begin{aligned}
G_{i} & =8 e^{-3 i \zeta_{0} / 2} \sin \left(\zeta_{0}\right) \sin \left(\zeta_{1}\right) \sin \left(\zeta_{2}\right) X_{i}, \\
G_{i}^{-1} & =8 e^{3 i \zeta_{0} / 2} \sin \left(\zeta_{0}\right) \sin \left(\zeta_{1}\right) \sin \left(\zeta_{2}\right) X_{i}^{t} \\
E_{i} & =X_{i}\left(\zeta_{0}\right)
\end{aligned}
$$

The normalization is taken so that $G_{i}^{-1}$ will be the inverse of $G_{i}$,

$$
G_{i} G_{i}^{-1}=1_{i}
$$

in view of the inversion relation eq. (1.22).

We can now express the projection operators $P_{i}^{a}$ in terms of $G_{i}, G_{i}^{-1}$ and $E_{i}$. This is given by solving the set of equations which are obtained from the ansatz for the Boltzmann weights, eqs. (1.20)-(1.21),

$$
\begin{aligned}
G_{i}= & i e^{-\frac{5}{2} i \zeta_{0}-i \zeta_{1}-i \zeta_{2}}\left(e^{2 i \zeta_{0}} P_{i}^{1}-e^{2 i \zeta_{0}+2 i \zeta_{1}} P_{i}^{2}+e^{2 i \zeta_{0}+2 i \zeta_{1}+2 i \zeta_{2}} P_{i}^{3}-P_{i}^{0}\right), \\
G_{i}^{-1}= & i e^{\frac{1}{2} i \zeta_{0}-i \zeta_{1}-i \zeta_{2}}\left(e^{2 i \zeta_{0}+2 i \zeta_{1}+2 i \zeta_{2}} P_{i}^{0}-e^{2 i \zeta_{1}+2 i \zeta_{2}} P_{i}^{1}+e^{2 i \zeta_{2}} P_{i}^{2}-P_{i}^{3}\right), \\
E_{i}= & \frac{e^{-3 i \zeta_{0}}\left(1+e^{2 i \zeta_{0}}\right)\left(-1+e^{i \zeta_{0}+i \zeta_{1}}\right)\left(1+e^{i \zeta_{0}+i \zeta_{1}}\right)}{\left(-1+e^{i \zeta_{1}}\right)\left(1+e^{i \zeta_{1}}\right)\left(-1+e^{i \zeta_{2}}\right)\left(1+e^{i \zeta_{2}}\right)} \\
& \times\left(-1+e^{i \zeta_{0}+i \zeta_{2}}\right)\left(1+e^{i \zeta_{0}+i \zeta_{2}}\right) P_{i}^{0},
\end{aligned}
$$

along with

$$
\sum_{a=0}^{3} P_{i}^{a}=1 .
$$

Our purpose is to describe the algebra obeyed by $G_{i}, G_{i}^{-1}$ and $E_{i}$. These are defined by

$$
\begin{aligned}
G_{i} & =8\left[\prod_{r=0}^{2} \sin \left(\zeta_{r}\right)\right] e^{-3 i \lambda / 2} X_{i}, \\
G_{i}^{-1} & =8\left[\prod_{r=0}^{2} \sin \left(\zeta_{r}\right)\right] e^{3 i \lambda / 2} X_{i}^{t}, \\
E_{i} & =X_{i}(\lambda) .
\end{aligned}
$$

By slight abusing the notations, we also call this algebra 4-CB (conformal braiding) algebra.

Due to the inversion relation, eq. (1.22) we have the relation,

$$
G_{i} G_{i}^{-1}=1_{i}
$$


The phase in eq. (3.8) is arbitrary and is set to simplify the 4-CB algebra. We have also the braiding relation,

$$
G_{i} G_{i+1} G_{i}=G_{i+1} G_{i} G_{i+1}, \quad G_{i} G_{j}=G_{j} G_{i} \text { if }|i-j| \geq 2 .
$$

We already know that $E_{i}$ obeys the Temperley-Lieb algebra, eqs. (1.26)-(1.27),

$$
E_{i} E_{i \pm 1} E_{i}=E_{i}, \quad E_{i}^{2}=b E_{i}, \quad \text { where } b=\prod_{r=0}^{2} \frac{\sin \left(\lambda+\zeta_{r}\right)}{\sin \left(\zeta_{r}\right)} .
$$

The next relations are,

$$
G_{i} E_{i}=E_{i} G_{i}=l^{-1} E_{i},
$$

which follow by taking the ansatz eqs. (1.20)-(1.21) for $G_{i}$ and using the projection relations, eq. (1.18), $P_{i}^{a} P_{i}^{0}=P_{i}^{0} P_{i}^{a}=\delta_{a, 0} P_{i}^{0}$. The value of $l$ is,

$$
l=i e^{i\left(3 \lambda / 2+\zeta_{0}+\zeta_{1}+\zeta_{2}\right)} .
$$

We can calculate,

$$
G_{i}^{2}=-e^{-5 i \zeta_{0}-2 i \zeta_{1}-2 i \zeta_{2}} P_{i}^{0}-e^{-i \zeta_{0}-2 i \zeta_{1}-2 i \zeta_{2}} P_{i}^{1}-e^{-i \zeta_{0}+2 i \zeta_{1}-2 i \zeta_{2}} P_{i}^{2}-e^{-i \zeta_{0}+2 i \zeta_{1}+2 i \zeta_{2}} P_{i}^{3} .
$$

We can substitute the expressions for $P_{i}^{a}$ from $G_{i}, G_{i}^{-1}$ and $E_{i}$, eqs. (3.4)-(3.6). We then find the relation expressing $G_{i}^{2}$,

$$
\begin{aligned}
G_{i}^{2}= & i e^{-\frac{1}{2} i \zeta_{0}-i \zeta_{1}-i \zeta_{2}}\left(1-e^{2 i \zeta_{1}}+e^{2 i \zeta_{1}+2 i \zeta_{2}}\right) G_{i}+i e^{-\frac{3}{2} i \zeta_{0}+i \zeta_{1}-i \zeta_{2}} G_{i}^{-1} \\
& +\frac{e^{-2 i \zeta_{0}-2 i \zeta_{1}-2 i \zeta_{2}}\left(e^{2 i \zeta_{1}}-1\right)\left(1+e^{2 i \zeta_{0}+2 i \zeta_{1}+2 i \zeta_{2}}\right)\left(e^{2 i \zeta_{2}}-1\right)}{\left(e^{2 i \zeta_{0}+2 i \zeta_{2}}-1\right)} E_{i} \\
& -e^{-i \zeta_{0}-2 i \zeta_{2}}\left(1-e^{2 i \zeta_{2}}+e^{2 i \zeta_{1}+2 i \zeta_{2}}\right) .
\end{aligned}
$$

We define the coefficients $\alpha, \beta, \gamma$ and $\delta$ by writing this equation as

$$
G_{i}^{2}=\alpha+\beta E_{i}+\gamma G_{i}+\delta G_{i}^{-1}
$$

This is the skein relation.

From the skein relation, eq. (3.16), we find the relation,

$$
G_{i \pm 1} G_{i} E_{i \pm 1}=E_{i} G_{i \pm 1} G_{i}
$$

which follows by expressing $E_{i}=\left(G_{i}^{2}-\alpha-\gamma G_{i}-\delta G_{i}^{-1}\right) / \beta$ from the skein relation eq. (3.16) and inserting it into eq. (3.10).

The additional relations areconjectural, but hold in all examples we have checked. These relations assume the same form as the Birman-Murakami-Wenzl (BMW) algebra [6, 7] and are summarized below.

$$
\begin{aligned}
G_{i \pm 1} G_{i} E_{i \pm 1} & =E_{i} E_{i \pm 1}, \quad G_{i \pm 1} E_{i} G_{i \pm 1}=G_{i}^{-1} E_{i \pm 1} G_{i}^{-1}, \\
G_{i \pm 1} E_{i} E_{i \pm 1} & =G_{i}^{-1} E_{i \pm 1}, \quad E_{i \pm 1} E_{i} G_{i \pm 1}=E_{i \pm 1} G_{i}^{-1}, \\
E_{i} G_{i \pm 1} E_{i} & =l E_{i} .
\end{aligned}
$$


The last relation follows from the Yang Baxter equation, see section 1. It is

$$
g(i, i+1, i)=g(i+1, i, i+1)
$$

where

$$
\begin{aligned}
g= & a_{1,2,4}+a_{1,3,1}+a_{4,2,1}+i q^{-\zeta_{0} / 2+\zeta_{1}-\zeta_{2}}\left(a_{1,3,4}+a_{4,2,4}+a_{4,3,1}\right) \\
& +i q^{\zeta_{0} / 2-\zeta_{1}+\zeta_{2}}\left(a_{2,3,4}+a_{4,1,4}+a_{4,3,2}\right) \\
& +i \frac{q^{\zeta_{1}+\zeta_{2}}}{\left(q^{2 \zeta_{1}}-1\right)\left(q^{2 \zeta_{2}}-1\right)}\left(q^{\zeta_{0} / 2} a_{1,2,1}+q^{-\zeta_{0} / 2} a_{2,1,2}\right)+z a_{4,3,4}
\end{aligned}
$$

where

$$
\begin{aligned}
z= & \frac{q^{-\zeta_{0}-2 \zeta_{1}-2 \zeta_{2}}\left(q^{2 \zeta_{1}}-1\right)\left(q^{2 \zeta_{2}}-1\right)}{q^{2 \zeta_{0}+2 \zeta_{2}-1}} \\
& \times\left(2 q^{2 \zeta_{0}+2 \zeta_{2}}+2 q^{2 \zeta_{0}+2 \zeta_{1}+2 \zeta_{2}}+q^{4 \zeta_{0}+2 \zeta_{1}+4 \zeta_{2}}+1\right) .
\end{aligned}
$$

We denoted by $a_{i, j, k}(r, s, t)$ the element of the algebra $a_{i}[r] a_{j}[s] a_{k}[t]$ where $a_{i}[r]$ is $G_{r}, G_{r}^{-1}, E_{r}$ or $1_{r}$, if $i=1,2,3,4$, respectively.

This summarizes all the relations of the 4-CB algebra. As we will show below, these are all the relations that follow from the YBE and are equivalent to it. We checked these relations for the model $\mathrm{SU}(2)$ fused $3 \times 3$ as described in ref. [3]. We also checked these relations for the $G_{2}$ model described in section 5 for $q=0.7$. We find a complete agreement with the 4-CB algebra. In section 5 we will prove this algebra for $G_{2}$.

\section{1 $\boldsymbol{n}=4 \mathrm{YBE}$ and relations $(3.11)-(3.21)$}

Our goal now is to verify that all the relations (3.11)-(3.21), represent a certain solution of the YBE, if(f) the parameters are fixed in appropriate way. The idea of the check is rather simple and follows closely the analogous consideration for 3-blocks case, described in the previous section.

Denoting $s_{0}=e^{i \zeta_{0}}, s_{1}=e^{i \zeta_{1}}, s_{2}=e^{i \zeta_{2}}$, we get the projectors

$$
\begin{aligned}
P_{i}^{0}= & \frac{s_{0}^{3}\left(s_{1}^{2}-1\right)\left(s_{2}^{2}-1\right)}{\left(s_{0}^{2}+1\right)\left(s_{0}^{2} s_{1}^{2}-1\right)\left(s_{0}^{2} s_{2}^{2}-1\right)} E_{i}, \\
P_{i}^{1}= & \frac{i s_{2} s_{1}^{3}}{\sqrt{s_{0}}\left(s_{1}^{2}+1\right)\left(s_{1}^{2} s_{2}^{2}-1\right)} G_{i}^{-1}+\frac{i \sqrt{s_{0}} s_{2} s_{1}}{\left(s_{1}^{2}+1\right)\left(s_{1}^{2} s_{2}^{2}-1\right)} G_{i}+ \\
& +\frac{s_{0}\left(s_{1}^{2}-1\right)\left(s_{2}^{2}-1\right)\left(s_{0}^{2} s_{1}^{2} s_{2}^{2}+1\right)}{\left(s_{0}^{2}+1\right)\left(s_{1}^{2}+1\right)\left(s_{0}^{2} s_{2}^{2}-1\right)\left(s_{1}^{2} s_{2}^{2}-1\right)} E_{i}+\frac{\left(s_{2}^{2}-1\right) s_{1}^{2}}{\left(s_{1}^{2}+1\right)\left(s_{1}^{2} s_{2}^{2}-1\right)} 1_{i}, \\
P_{i}^{2}= & \frac{i \sqrt{s_{0}} s_{1} s_{2}}{\left(s_{1}^{2}+1\right)\left(s_{2}^{2}+1\right)} G_{i}-\frac{i s_{1} s_{2}}{\sqrt{s_{0}}\left(s_{1}^{2}+1\right)\left(s_{2}^{2}+1\right)} G_{i}^{-1}- \\
& -\frac{s_{0}\left(s_{1}^{2}-1\right)\left(s_{2}^{2}-1\right)\left(s_{0}^{2} s_{1}^{2} s_{2}^{2}+1\right)}{\left(s_{0}^{2} s_{1}^{2}-1\right)\left(s_{1}^{2}+1\right)\left(s_{0}^{2} s_{2}^{2}-1\right)\left(s_{2}^{2}+1\right)} E_{i}+\frac{\left(s_{1}^{2} s_{2}^{2}+1\right)}{\left(s_{1}^{2}+1\right)\left(s_{2}^{2}+1\right)} 1_{i},
\end{aligned}
$$

where (3.7) is used in order to eliminate $P_{i}^{3}$. Note that the skein relation (3.16) allows also to use other choices of any three independent parameters. 
Taking into account the explicit form of the conjectured trigonometric solution eqs. (1.20)-(1.21), the Yang Baxter equation (1.5) can be written in the form

$$
Y B E \Rightarrow \sum_{r, s} C_{r s}^{Y B E} q_{1}^{r} q_{2}^{s}=0
$$

where $q_{1}=e^{i u}, q_{2}=e^{i v}$, the integers $r, s$ are in the region $[0,2 n]$, and the coefficients $C_{r s}^{Y B E}$ are expressed in terms of the generators $E_{i}, G_{i}, G_{i}^{-1}$ and $1_{i}$. Hence, in order to fulfil the YBE for generic values of the spectral parameters $u, v$ one has to ensure that the coefficients $C_{r s}^{Y B E}=0$, for all $r$ and $s$.

In the 4 -blocks case simple counting shows that there are 37 relations, $r \in[0,8]$ step 2 , and $s \in[\operatorname{Max}(r-6,0), \operatorname{Min}(r+6,8)]$ step 2. Taking into account the factorised form of the coefficients $f_{a}(u)$ in terms of sine functions, see eq. (1.21), we note that for general $n$ the number of the relations is given by Hex number, i. e. by the number of partitions of $6(n-1)$ into at most 3 parts, which is $N(n)=3(n-1) n+1$, so that for lower values of $n$ we have $1,7,19,37,61,91, \ldots$.

In our case a few relations are written below explicitly

$$
\begin{aligned}
& (r, s)=(0,0): \quad a_{1,1,1}(i, i+1, i)-a_{1,1,1}(i+1, i, i+1)=0, \\
& (r, s)=(0,2): \quad s_{1}^{2} s_{2}^{2} s_{0}^{3 / 2} a_{4,1,1}(i+1, i, i+1)-s_{2}^{2} s_{0}^{3 / 2} a_{4,1,1}(i+1, i, i+1) \\
& +s_{2}^{2} s_{0}^{3 / 2} a_{1,1,4}(i, i+1, i)+s_{1}^{2} s_{0}^{3 / 2} a_{1,1,4}(i, i+1, i)-s_{1}^{2} s_{0}^{3 / 2} a_{4,1,1}(i+1, i, i+1) \\
& -s_{1}^{2} s_{2}^{2} s_{0}^{3 / 2} a_{1,1,4}(i, i+1, i)-s_{0}^{3 / 2} a_{1,1,4}(i, i+1, i)+s_{0}^{3 / 2} a_{4,1,1}(i+1, i, i+1) \\
& -i s_{1} s_{2} s_{0}^{2} a_{1,1,1}(i, i+1, i)+i s_{1} s_{2} s_{0}^{2} a_{1,1,1}(i+1, i, i+1)+i s_{1} s_{2} s_{0} a_{1,1,2}(i, i+1, i) \\
& -i s_{1} s_{2} s_{0} a_{2,1,1}(i+1, i, i+1)-s_{1}^{2} \sqrt{s_{0}} a_{1,1,3}(i, i+1, i)+s_{1}^{2} s_{2}^{2} \sqrt{s_{0}} a_{1,1,3}(i, i+1, i) \\
& -s_{2}^{2} \sqrt{s_{0}} a_{1,1,3}(i, i+1, i)+s_{1}^{2} \sqrt{s_{0}} a_{3,1,1}(i+1, i, i+1)-\sqrt{s_{0}} a_{3,1,1}(i+1, i, i+1) \\
& -i s_{1} s_{2} a_{1,1,1}(i, i+1, i)+i s_{1} s_{2} a_{1,1,1}(i+1, i, i+1)+\sqrt{s_{0}} a_{1,1,3}(i, i+1, i) \\
& -s_{1}^{2} s_{2}^{2} \sqrt{s_{0}} a_{3,1,1}(i+1, i, i+1)+s_{2}^{2} \sqrt{s_{0}} a_{3,1,1}(i+1, i, i+1)=0, \\
& (r, s)=(0,4): \quad s_{1}^{2} s_{2}^{2} s_{0}^{3 / 2} a_{3,1,1}(i+1, i, i+1)-s_{2}^{2} s_{0}^{3 / 2} a_{3,1,1}(i+1, i, i+1) \\
& +s_{2}^{2} s_{0}^{3 / 2} a_{1,1,3}(i, i+1, i)-s_{0}^{3 / 2} a_{1,1,3}(i, i+1, i)-s_{1}^{2} s_{0}^{3 / 2} a_{3,1,1}(i+1, i, i+1) \\
& +s_{0}^{3 / 2} a_{3,1,1}(i+1, i, i+1)+s_{1}^{2} s_{0}^{3 / 2} a_{1,1,3}(i, i+1, i)-s_{1}^{2} s_{2}^{2} s_{0}^{3 / 2} a_{1,1,3}(i, i+1, i) \\
& -i s_{1} s_{2} s_{0}^{2} a_{1,1,2}(i, i+1, i)+i s_{1} s_{2} s_{0}^{2} a_{2,1,1}(i+1, i, i+1)+i s_{1} s_{2} s_{0} a_{1,1,1}(i, i+1, i) \\
& -i s_{1} s_{2} s_{0} a_{1,1,1}(i+1, i, i+1)-s_{1}^{2} \sqrt{s_{0}} a_{1,1,4}(i, i+1, i)+s_{1}^{2} s_{2}^{2} \sqrt{s_{0}} a_{1,1,4}(i, i+1, i) \\
& -s_{2}^{2} \sqrt{s_{0}} a_{1,1,4}(i, i+1, i)+\sqrt{s_{0}} a_{1,1,4}(i, i+1, i)+s_{1}^{2} \sqrt{s_{0}} a_{4,1,1}(i+1, i, i+1) \\
& -i s_{1} s_{2} a_{1,1,2}(i, i+1, i)+i s_{1} s_{2} a_{2,1,1}(i+1, i, i+1)-\sqrt{s_{0}} a_{4,1,1}(i+1, i, i+1) \\
& -s_{1}^{2} s_{2}^{2} \sqrt{s_{0}} a_{4,1,1}(i+1, i, i+1)+s_{2}^{2} \sqrt{s_{0}} a_{4,1,1}(i+1, i, i+1)=0, \\
& (r, s)=(0,6): \quad a_{1,1,2}(i, i+1, i)-a_{2,1,1}(i+1, i, i+1)=0,
\end{aligned}
$$

Here we use the notation introduced just below eq. (3.21). We do not write all the relations since their expressions are bulky in general, while the computation is rather straightforward. 
Our next task is to implement the relations (3.11)-(3.19). We perform this gradually. First, we use the "simple" parameter-free relations, like the braiding relations (3.10), parameter-free Temperley-Lieb algebra relations (3.11), the relation (3.18), and all the relations, which follow from these relations. We chose this order not to end up with some particular solution of the YBE, obeying softer algebraic constraints, but existing only for special choices of the parameters. The relations are

$$
\begin{array}{ll}
a_{1,1,1}(i+1, i, i+1)=a_{1,1,1}(i, i+1, i), & a_{2,1,1}(i+1, i, i+1)=a_{1,1,2}(i, i+1, i), \\
a_{1,1,2}(i+1, i, i+1)=a_{2,1,1}(i, i+1, i), & a_{2,2,1}(i+1, i, i+1)=a_{1,2,2}(i, i+1, i), \\
a_{1,2,2}(i+1, i, i+1)=a_{2,2,1}(i, i+1, i), & a_{2,2,2}(i+1, i, i+1)=a_{2,2,2}(i, i+1, i), \\
a_{3,1,1}(i+1, i, i+1)=a_{1,1,3}(i, i+1, i), & a_{1,1,3}(i+1, i, i+1)=a_{3,1,1}(i, i+1, i), \\
a_{2,3,1}(i+1, i, i+1)=a_{1,3,2}(i, i+1, i), & a_{2,4,1}(i+1, i, i+1)=a_{1,4,2}(i, i+1, i), \\
a_{1,3,2}(i+1, i, i+1)=a_{2,3,1}(i, i+1, i), & a_{1,4,2}(i+1, i, i+1)=a_{2,4,1}(i, i+1, i), \\
a_{2,2,3}(i+1, i, i+1)=a_{3,2,2}(i, i+1, i), & a_{3,2,2}(i+1, i, i+1)=a_{2,2,3}(i, i+1, i) .
\end{array}
$$

For example, to obtain the last relation $a_{3,2,2}(i+1, i, i+1)=a_{2,2,3}(i, i+1, i)$, which is explicitly $E_{i+1} G_{i}^{-1} G_{i+1}^{-1}=G_{i}^{-1} G_{i+1}^{-1} E_{i}$, we multiply the equation (3.17) from both sides by $G_{i}^{-1} G_{i+1}^{-1}$ and then use the inversion relation (3.9).

Now we are in the position to implement the relations involving parameters (like skein relation, etc.) and also all their derivatives. In principle, this can be done in deferent ways, our guiding principle is to exclude all linear dependent elements, simultaneously choosing among the linear independent elements those with the maximal numbers of $1_{i}$ generators. First set of the relations is

$$
\begin{aligned}
& a_{1,2,3}(x)=\alpha a_{4,3,3}(x)+\beta a_{3,3,3}(x)+\gamma a_{1,3,3}(x)+\delta a_{2,3,3}(x), \\
& a_{2,1,3}(x)=-\frac{\beta l a_{3,3,3}(x)}{\delta}-\frac{\alpha a_{2,3,3}(x)}{\delta}-\frac{\gamma a_{4,3,3}(x)}{\delta}+\frac{a_{1,3,3}(x)}{\delta}, \\
& a_{3,2,1}(x)=\alpha a_{3,3,4}(x)+\beta a_{3,3,3}(x)+\gamma a_{3,3,1}(x)+\delta a_{3,3,2}(x), \\
& a_{3,1,2}(x)=-\frac{\beta l a_{3,3,3}(x)}{\delta}-\frac{\alpha a_{3,3,2}(x)}{\delta}-\frac{\gamma a_{3,3,4}(x)}{\delta}+\frac{a_{3,3,1}(x)}{\delta},
\end{aligned}
$$

which follow from the skein relation. Here $x$ stands for either $(i+1, i, i+1)$ or $(i, i+1, i)$. For example, in order to obtain the first one we use the relation $G_{i+1}^{-1} E_{i}=G_{i} E_{i+1} E_{i}$ from eq. (3.18), and then the 4 -blocks skein relation to exclude $G_{i}^{2}$ on the first place.

Similarly, we get

$$
\begin{aligned}
a_{3,1,3}(i, i+1, i) & =l a_{4,3,4}(i+1, i, i+1), & a_{3,1,3}(i+1, i, i+1) & =l a_{4,3,4}(i, i+1, i), \\
a_{2,4,3}(i+1, i, i+1) & =l a_{4,3,4}(i, i+1, i), & a_{2,4,3}(i, i+1, i) & =l a_{4,3,4}(i+1, i, i+1), \\
a_{3,4,2}(i+1, i, i+1) & =l a_{4,3,4}(i, i+1, i), & a_{3,4,2}(i, i+1, i) & =l a_{4,3,4}(i+1, i, i+1), \\
a_{3,2,3}(i, i+1, i) & =\frac{a_{4,3,4}(i+1, i, i+1)}{l}, & a_{3,2,3}(i+1, i, i+1) & =\frac{a_{4,3,4}(i, i+1, i)}{l} \\
a_{1,4,3}(i+1, i, i+1) & =\frac{a_{4,3,4}(i, i+1, i)}{l}, & a_{1,4,3}(i, i+1, i) & =\frac{a_{4,3,4}(i+1, i, i+1)}{l}, \\
a_{3,4,1}(i+1, i, i+1) & =\frac{a_{4,3,4}(i, i+1, i)}{l}, & a_{3,4,1}(i, i+1, i) & =\frac{a_{4,3,4}(i+1, i, i+1)}{l} .
\end{aligned}
$$


In particular, the first two correspond to the last equation in (3.18) and others can be derived from it.

We also have

$$
\begin{aligned}
a_{1,4,1}(i \pm 1, i, i \pm 1)= & \alpha a_{4,4,4}(i, i \pm 1, i)+\beta a_{4,3,4}(i, i \pm 1, i) \\
& +\gamma a_{4,1,4}(i, i \pm 1, i)+\delta a_{4,2,4}(i, i \pm 1, i) \\
a_{2,4,2}(i \pm 1, i, i \pm 1)= & \frac{a_{4,1,4}(i, i \pm 1, i)}{\delta}-\frac{\beta l a_{4,3,4}(i, i \pm 1, i)}{\delta} \\
& -\frac{\alpha a_{4,2,4}(i, i \pm 1, i)}{\delta}-\frac{\gamma a_{4,4,4}(i, i \pm 1, i)}{\delta}
\end{aligned}
$$

and

$$
a_{3,4,3}(i \pm 1, i, i \pm 1)=\left(\frac{1}{\beta l^{2}}-\frac{\alpha}{\beta}-\frac{\gamma}{\beta l}-\frac{\delta l}{\beta}\right) a_{4,3,4}(i, i \pm 1, i)
$$

which follow again from the skein relation, and from the relation (3.12).

And also some trivial relations involving unity operator, like

$$
\begin{aligned}
a_{2,4,1}(i, i+1, i) & =a_{2,4,1}(i+1, i, i+1), \\
a_{1,4,2}(i, i+1, i) & =a_{2,4,1}(i, i+1, i), \\
a_{1,4,2}(i+1, i, i+1) & =a_{2,4,1}(i+1, i, i+1), \\
a_{k, s, 4}(i+1, i, i+1) & =a_{4, k, s}(i, i+1, i), \\
a_{4, k, s}(i+1, i, i+1) & =a_{k, s, 4}(i, i+1, i), \\
a_{k, 4,4}(i, i+1, i) & =a_{4, k, 4}(i+1, i, i+1), \\
a_{4,4, k}(i, i+1, i) & =a_{4, k, 4}(i+1, i, i+1), \\
a_{k, 4,4}(i+1, i, i+1) & =a_{4, k, 4}(i, i+1, i), \\
a_{4,4, k}(i+1, i, i+1) & =a_{4, k, 4}(i, i+1, i),
\end{aligned}
$$

which are valid for any $k, s$. For example, the first relation is $1_{i} 1_{i+1}=1_{i+1} 1_{i}$, which is obviously true.

Finally, we use

$$
\begin{aligned}
l & =i q^{\frac{5 \zeta_{0}}{2}+\zeta_{1}+\zeta_{2}} \\
\alpha & =-q^{-\zeta_{0}-2 \zeta_{2}}\left(-q^{2 \zeta_{2}}+q^{2 \zeta_{1}+2 \zeta_{2}}+1\right) \\
\beta & =\frac{q^{-2 \zeta_{0}-2 \zeta_{1}-2 \zeta_{2}}\left(q^{2 \zeta_{1}}-1\right)\left(q^{2 \zeta_{2}}-1\right)\left(q^{2 \zeta_{0}+2 \zeta_{1}+2 \zeta_{2}}+1\right)}{q^{2 \zeta_{0}+2 \zeta_{2}}-1} \\
\gamma & =i q^{-\frac{\zeta_{0}}{2}-\zeta_{1}-\zeta_{2}}\left(-q^{2 \zeta_{1}}+q^{2 \zeta_{1}+2 \zeta_{2}}+1\right) \\
\delta & =i q^{-\frac{3}{2} \zeta_{0}+\zeta_{1}-\zeta_{2}}
\end{aligned}
$$


or in terms of $s_{1}, s_{2}, s_{3}$,

$$
\begin{aligned}
& l=i s_{0}^{5 / 2} s_{1} s_{2} \\
& \alpha=-\frac{s_{1}^{2} s_{2}^{2}-s_{2}^{2}+1}{s_{0} s_{2}^{2}} \\
& \beta=\frac{\left(s_{1}^{2}-1\right)\left(s_{2}^{2}-1\right)\left(s_{0}^{2} s_{1}^{2} s_{2}^{2}+1\right)}{s_{0}^{2} s_{1}^{2} s_{2}^{2}\left(s_{0}^{2} s_{2}^{2}-1\right)} \\
& \gamma=\frac{i\left(s_{2}^{2} s_{1}^{2}-s_{1}^{2}+1\right)}{\sqrt{s_{0}} s_{1} s_{2}} \\
& \delta=\frac{i s_{1}}{s_{0}^{3 / 2} s_{2}}
\end{aligned}
$$

Substituting the relations (3.14)-(3.21) into 37 relations obtained from the YBE, eq. (3.24), we find that some of the YBE relations become fulfilled, and we are left with 19 relations. For these remaining relations we find that they are compatible if and only if the relation $g(i, i+1, i)=g(i+1, i, i+1)$, eqs. (3.19)-(3.20), is imposed.

\section{Three parameter link invariant}

Let $B M W_{n}^{\prime}$ be the 4-CB algebra described in section 3 with generators $1, G_{1}, \ldots, G_{n-1}$ and also $E_{i}, i=1,2, \ldots, n-1$. Let $b$ be as in eq. (3.11) and $l$ as in eq. (3.13). We conjecture the existence of a unique trace function

$$
\tau: \bigcup B M W_{n}^{\prime} \rightarrow \mathbf{C}
$$

which satisfies the following properties

1) $\tau(a+b)=\tau(a)+\tau(b)$.

2) $\tau(a b)=\tau(b a)$.

3) $\tau(1)=1$ and $\tau\left(E_{i}\right)=b$.

4) $\tau\left(w \cdot G_{n}\right)=l^{-1} \tau(w)$, and $\tau\left(w \cdot G_{n}^{-1}\right)=l \tau(w)$, where $w \in B M W_{n}^{\prime}$.

The existence of such trace will allow us to define a link invariant extending the results of ref. [3]. We do it in the following way. We assign $E_{i}$ and $G_{i}$ to diagrams, as in figure 1. Given a composite diagram, we assign it an expression in terms of $E_{i}$ and $G_{i}$ in the expected way, and apply $\tau$ to the result. We denote by $L(D)$ the result of this assignment, performed on a diagram $D$.

From the properties of $\tau$ and the relations of $B M W_{n}^{\prime}$ one can show that $L$ has the following properties:

1) $L(O)=b$,

2) $L\left(S_{r}\right)=l^{-1} L(S)$ and $L\left(S_{l}\right)=l L(S)$, 


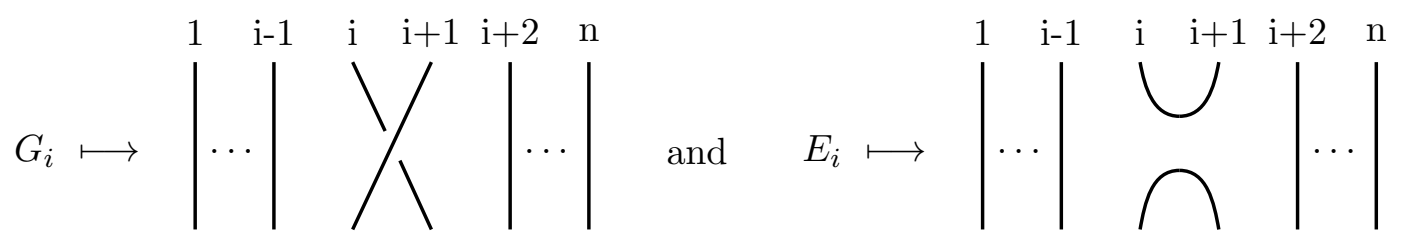

Figure 1. The isomorphism between 4-CB and the tangle algebra.

Type I

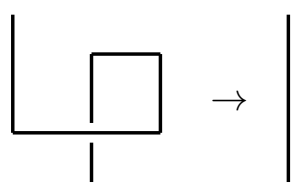

Type II

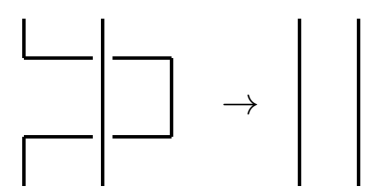

Type III

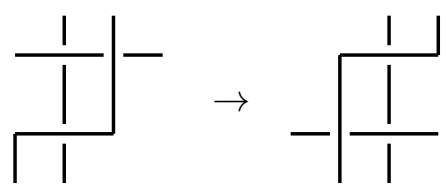

Figure 2. Reidemeister moves.

3) $L$ is unchanged by Reidemeister moves II,III, which are described in figure 2 .

Here $O$ is the standard diagram for the unknot, $S$ is a strand and $S_{r}$ (respectively $S_{l}$ ) is the same strand with a right handed curl (respectively left handed) as in type I Reidemeister move. The third property follows from the relations of the 4-CB algebra.

We correct $L$ to form a Markov trace by putting

$$
\nu(K)=l^{\omega(K)} L(K),
$$

where $K$ is link, $\nu(K)$ is the link invariant, $\omega(K)$ is the writhe of the link defined as the number of left crossings minus the number of right crossings. By properties 2),3) above we see that $\nu$ is a link invariant.

If the conjecture is correct then the resulting link invariant belongs to a three parameter family of invariants, where the parameters are the $\zeta_{i}$ s. In section 5 , we will show that the link invariant and the 4-CB algebra follow for the case of $G_{2}$.

\section{$5 \quad G_{2}$ IRF model}

We wish to check our conjecture for the IRF Boltzmann weights, eqs. (1.20)-(1.21), for the $G_{2}$ IRF model. The explicit Boltzmann weights of this model were given by Kuniba et al. [16]. We wish to check our general ansatz, eqs. (1.20)-(1.21), specialized to the $G_{2}$ case. The model is defined by taking for the RCFT $\mathcal{O}$ the WZW model based on the Lie algebra $G_{2}$ at level $k$. For the field $h=v$ we substitute the fundamental representation [7], which is the 7 dimensional representation. Thus the model is $\operatorname{IRF}\left(G_{2},[7],[7]\right)$. In the fusion product of $[h]$ and $[v]$, eq. (1.11), we encounter four representations,

$$
[7] \times[7]=[1]+[14]+[27]+[7] .
$$

Thus, the $G_{2}$ theory is a four blocks theory. Note that we chose this order for the fields appearing in the product, $\psi_{0}=[1], \psi_{1}=[14], \psi_{2}=[27]$ and $\psi_{3}=[7]$, to be consistent with the Boltzmann weights of Kuniba et al. 
The dimensions of the fields in a WZW theory are given by

$$
\Delta_{\lambda}=\frac{\lambda(\lambda+2 \rho)}{2(k+g)}
$$

where $\lambda$ is the highest weight of the representation, $\rho$ is half the sum of positive roots, and $g$ is the dual Coxeter number.

We can now compute the crossing parameters $\zeta_{i}$, using eq. (1.19). We find for the dimensions of the fields $\psi_{i}$,

$$
\Delta_{0}=0, \quad \Delta_{1}=\frac{12}{k+4}, \quad \Delta_{2}=\frac{14}{k+4}, \quad \Delta_{3}=\frac{6}{k+4},
$$

where $\Delta_{i}$ is the dimension of the field $\psi_{i}$.

The crossing parameters are given, by eq. (1.19), $\zeta_{i}=\pi\left(\Delta_{i+1}-\Delta_{i}\right) / 2$,

$$
\zeta_{0}=\lambda=-\frac{6 \pi}{k+4}, \quad \zeta_{1}=-\frac{\pi}{k+4}, \quad \zeta_{2}=\frac{4 \pi}{k+4} .
$$

Note that we inverted the signs of the crossing parameters. This is always allowed since in the ansatz, eqs. (1.20)-(1.21), we can invert the signs of the crossing parameters along with the sign of $u$ and the resulting Boltzmann weight is not changed (up to a possible sign).

To calculate the Boltzmann weight $X_{i}(u)$ we need to know the braiding matrices of the RCFT $\mathcal{O}$. Unfortunately, this has never been calculated directly. So our idea is to extract the braiding matrices from the solution of Kuniba et al., and then to compare that $X_{i}(u)$, as given by eqs. (1.20)-(1.21) agrees with the Boltzmann weights of Kuniba et al. This will assure that our conjecture is correct for this theory.

We extract the projection operators $P_{i}^{a}$ from the above eqs. (3.4)-(3.6) and insert them into our ansatz of the Boltzmann weights, eqs. (1.20)-(1.21). Now we are in a position to compare our Boltzmann weights with those of Kuniba et al. We preform this calculation numerically by choosing $q=e^{i \pi /(k+4)}=0.7$. We compare all the Boltzmann weights for an arbitrary spectral parameter, $u$. We find a complete agreement. This illustrates that our ansatz is correct for $G_{2}$ theory.

\subsection{Kuperberg's $G_{2}$ link invariant}

In ref. [8], Kuperberg introduced a tangle algebra for the $G_{2}$ link invariant. Our claim is that this link invariant is identical, for the special case of $G_{2}$, to our link invariant in section 4 . This proves the link invariant for this case, as well as the algebraic structure we find, namely the 4-CB algebra, for this case.

Recall from eq. (5.4) that the crossing multipliers for $G_{2}$ have the form

$$
\zeta_{0}=-\frac{6 \pi}{k+4}, \quad \zeta_{1}=-\frac{\pi}{k+4}, \quad \zeta_{2}=\frac{4 \pi}{k+4} .
$$

We find it convenient to define

$$
q=e^{i \pi /(k+4)} .
$$


Thus we find from eqs. (3.11)-(3.12) that the parameters of the algebra are given by

$$
l=i q^{-12}, \quad b=-\frac{[12][7][2]}{[6][4][1]},
$$

where we defined,

$$
[x]=q^{x}-q^{-x} .
$$

To make contact with Kuperberg's work we also rescale $G_{i}^{ \pm 1} \rightarrow i^{ \pm 1} G_{i}^{ \pm 1}$ and $E_{i} \rightarrow-E_{i}$. Also, his $q$ is our $q^{2}$. We then find from eqs. (3.15)-(3.16),

$$
G_{i}^{2}=-\left(-q^{14}+q^{12}-q^{10}+q^{6}-q^{4}+q^{2}\right) E_{i}+\left(q^{-2}+q^{4}-q^{6}\right)-\left(1-q^{-2}+q^{6}\right) G_{i}+q^{4} G_{i}^{-1} .
$$

Now, in Kalfagianni's work [9], the algebraic relations which follow from Kuperberg's tangle algebra were derived. This author finds exactly the 4 -CB algebra that we conjectured for the case of $G_{2}$, with exactly the parameters $l, b, \alpha, \beta, \gamma, \delta$ that we calculated from our general ansatz. This proves the 4-CB algebra for the case of $G_{2}$. It also shows the consistency of the link invariant, described in section 4 , for this special case.

Actually in ref. [9] additional relations are described. As will be shown in sections 6-7, these hold also for all 4-blocks theories.

\section{$6 \quad H$ and $K$ relations}

In Kuperberg paper [8], the diagrammatic operations $H$ and $K$ are defined. Kalfagianni [9] defined these algebraically. There it was shown that in the case of $G_{2}$ the operators $H$ and $K$ obey some far reaching algebraic relations. Our purpose here is to generalize Kalfagianni's algebra to all the 4-blocks lattice models. We will check these relations with the $\mathrm{SU}(2)$ fused $3 \times 3$ lattice models, assuming that if they hold both for $G_{2}$ and $\mathrm{SU}(2)$, they are correct generally. We prove these relations for any four blocks model in section 7 .

Our starting point are the relations, which hold for $G_{2}$,

$$
\begin{aligned}
G_{i}-q^{-1} G_{i}^{-1} & =\left(1-q^{-1}\right)\left(H_{i}+\left(q+q^{-1}\right) E_{i}-1\right), \\
G_{i}-q G_{i}^{-1} & =(1-q)\left(K_{i}-E_{i}+\left(q+q^{-1}\right)\right) .
\end{aligned}
$$

where $q=\exp \left[i \pi /(2(k+4)]\right.$, and $k$ is the level. If we substitute the expressions fo $G_{i}$ and $G_{i}^{-1}$ in accordance with our ansatz eqs. (1.20)-(1.21), we find that $H_{i}$ is proportional to $P_{i}^{3}$, the third projection operator. Our idea to generalize the operator $H_{i}$ by simply equating it with this projection operator

$$
H_{i}=P_{i}^{3}
$$

To express $H_{i}$ in terms of $G_{i}, G_{i}^{-1}, E_{i}$ and $1_{i}$ we simply solve the equations for them, eqs. (3.4)-(3.6), using the relation $P_{i}^{1}=1_{i}-P_{i}^{0}-P_{i}^{2}-P_{i}^{3}$, to find $P_{i}^{3}$. We thus get the 
relation,

$$
\begin{aligned}
z H_{i}= & \left(-e^{i \zeta(0)+2 i \zeta(2)}+e^{i \zeta(0)+2 i \zeta(1)+2 i \zeta(2)}+e^{i \zeta(0)+4 i \zeta(2)}-e^{i \zeta(0)+2 i \zeta(1)+4 i \zeta(2)}\right) E_{i} \\
& +\left(-e^{2 i \zeta(1)+2 i \zeta(2)}-e^{2 i \zeta(0)+4 i \zeta(2)}+e^{2 i \zeta(0)+2 i \zeta(1)+4 i \zeta(2)}+e^{2 i \zeta(2)}\right) 1_{i} \\
& +i e^{\frac{1}{2} i \zeta(0)+i \zeta(1)+3 i \zeta(2)} G_{i}-i e^{\frac{5}{2} i \zeta(0)+i \zeta(1)+5 i \zeta(2)} G_{i}+i e^{-\frac{1}{2} i \zeta(0)+i \zeta(1)+i \zeta(2)} G_{i}^{-1} \\
& -i e^{\frac{3}{2} i \zeta(0)+i \zeta(1)+3 i \zeta(2)} G_{i}^{-1}
\end{aligned}
$$

where $z$ is defined by

$$
z=\left(-1+e^{i \zeta(0)+i \zeta(2)}\right)\left(1+e^{i \zeta(0)+i \zeta(2)}\right)\left(-1+e^{2 i \zeta(1)+2 i \zeta(2)}\right)\left(1+e^{2 i \zeta(2)}\right)
$$

It is convenient to define the parameters $r_{j}$ as the coefficients of $H_{i}$,

$$
H_{i}=r_{1} 1_{i}+r_{2} E_{i}+r_{3} G_{i}+r_{4} G_{i}^{-1}
$$

where $H_{i}$ is given by eqs. (6.4)-(6.5) above. Note that $H_{i}$ is defined for any values of the crossing parameters $\zeta_{i}$ and thus for any four blocks theory.

We come now to the problem of defining $K_{i}$. We do this by imposing Kalfagianni's equation which holds for $G_{2}$,

$$
E_{i \pm 1} E_{i} H_{i \pm 1}=E_{i \pm 1} K_{i}
$$

and assuming that it holds for any theory and not just $G_{2}$. We substitute $H_{i}$ from eq. (6.4)(6.5) and use the relations of the BMW algebra eq. (3.18). We find that this relation holds if and only if $K_{i}$ has the expression,

$$
K_{i}=r_{2} 1_{i}+r_{1} E_{i}+r_{4} G_{i}+r_{3} G_{i}^{-1} .
$$

Again, this relation is general for any four blocks theory.

We are now in a position to check Kalfagianni's relations, which hold for $G_{2}$, to the other case that we investigated which is the $\mathrm{SU}(2)$ fused $3 \times 3$ model. We do this using the explicit Boltzmann weights which were given in ref. [3].

The crossing parameters for the $\mathrm{SU}(2) 3 \times 3$ model are given by [3]

$$
\zeta_{0}=\frac{\pi}{k+2}, \quad \zeta_{1}=\frac{2 \pi}{k+2}, \quad \zeta_{2}=\frac{3 \pi}{k+2}
$$

where $k$ is the level of the $\mathrm{SU}(2)$ model. 
We find that all of Kalfagianni relations hold also for $\mathrm{SU}(2)$ model. Below is a list of the 'simple' relations that hold not only for $G_{2}$ but also for $\mathrm{SU}(2)$.

$$
\begin{aligned}
H_{i} E_{i} & =0 \\
K_{i} E_{i} & =E_{i} K_{i}=d E_{i}, \\
K_{i} H_{i} & =H_{i} K_{i}=c H_{i}, \\
K_{i}^{2} & =a H_{i}+b K_{i}+e E_{i}+f, \\
H_{i} H_{j} & =H_{j} H_{i}, \text { if }|i-j| \geq 2, \\
K_{i} K_{j} & =K_{j} K_{i}, \text { if }|i-j| \geq 2, \\
G_{i \pm 1} G_{i} H_{i \pm 1} & =H_{i} G_{i \pm 1} G_{i}, \\
G_{i \pm 1} G_{i} K_{i \pm 1} & =K_{i} G_{i \pm 1} G_{i}, \\
E_{i} H_{i \pm 1} E_{i} & =x E_{i}, \\
E_{i} K_{i \pm 1} E_{i} & =0 \\
E_{i \pm 1} E_{i} H_{i \pm 1} & =E_{i \pm 1} K_{i}, \\
H_{i \pm 1} E_{i} E_{i \pm 1} & =K_{i \pm 1} E_{i}, \\
K_{i \pm 1} E_{i} E_{i \pm 1} & =H_{i \pm 1} E_{i}, \\
E_{i \pm 1} E_{i} K_{i \pm 1} & =E_{i \pm 1} H_{i}, \\
E_{i} H_{i \pm 1} H_{i} & =E_{i} K_{i \pm 1} K_{i} \\
H_{i \pm 1} H_{i} E_{i \pm 1} & =K_{i \pm 1} K_{i} E_{i \pm 1} \\
K_{i} E_{i \pm 1} H_{i} & =H_{i \pm 1} E_{i} K_{i \pm 1} \\
H_{i} E_{i \pm 1} H_{i} & =K_{i \pm 1} E_{i} K_{i \pm 1} .
\end{aligned}
$$

Some of these simple relations follow directly from the definition of $H_{i}$ and $K_{i}$ and the BMW algebra, but not all. Here $a, b, c, d, e, f$ and $x$ are some coefficients which can be easily computed from the definition eqs. (1.20)-(1.21).

We get now to the 'complicated' relations, following Kalfagianni. The relations are

$$
\begin{aligned}
H_{i} H_{i+1} H_{i}= & H_{i+1} H_{i} H_{i+1}-v_{1}\left(H_{i} E_{i+1} H_{i}-H_{i+1} E_{i} H_{i+1}\right) \\
& -v_{2}\left(H_{i} K_{i+1} H_{i}-H_{i+1} K_{i} H_{i+1}\right)-v_{3}\left(H_{i}-H_{i+1}\right) . \\
H_{i} H_{i \pm 1} K_{i}= & K_{i \pm 1} H_{i} H_{i \pm 1}-x_{1}\left(H_{i} K_{i \pm 1} H_{i}-H_{i \pm 1} K_{i} H_{i \pm 1}\right) \\
& -x_{2}\left(H_{i} E_{i \pm 1} H_{i}-H_{i \pm 1} E_{i} H_{i \pm 1}\right)-x_{3}\left(H_{i} K_{i \pm 1}-K_{i} H_{i \pm 1}\right) \\
& -x_{4}\left(K_{i \pm 1} E_{i}-E_{i \pm 1} K_{i}\right)-x_{5}\left(H_{i} E_{i \pm 1}-E_{i} H_{i \pm 1}\right)-x_{6}\left(H_{i}-H_{i \pm 1}\right) . \\
K_{i} K_{i+1} K_{i}= & K_{i+1} K_{i} K_{i+1}-z_{1}\left(H_{i} K_{i+1} H_{i}-H_{i+1} K_{i} H_{i+1}\right) \\
& -z_{2}\left(H_{i} K_{i+1}-H_{i+1} K_{i}\right)-z_{3}\left(K_{i+1} H_{i}-K_{i} H_{i+1}\right)-z_{4}\left(K_{i+1} E_{i}-K_{i} E_{i+1}\right) \\
& -z_{5}\left(E_{i} K_{i+1}-E_{i+1} K_{i}\right)-z_{6}\left(K_{i}-K_{i+1}\right) .
\end{aligned}
$$

As was shown by Kalfagianni, these equations hold for the $G_{2}$ model for some values of the parameters $v_{i}, x_{i}, z_{i}$. Our idea is to establish these relations for the $\mathrm{SU}(2) 3 \times 3$ model, for some values of the parameters. We substituted the Boltzmann weights and solved for 
the parameters using some configurations. We found that these relations are obeyed also for $\mathrm{SU}(2)$. For the parameters we find the following general relations:

$$
v_{1}=-r_{2}, \quad x_{2}=x_{3}=-r_{1}, \quad x_{4}=x_{6},
$$

where $r_{1}, r_{2}$ is given by eq. (6.6). We also find that the coefficients $z_{i}$ are the same as the coefficients $x_{i}$,

$$
z_{1}=x_{1}, \quad z_{2}=z_{3}=x_{2}=x_{3}, \quad z_{4}=z_{5}=x_{4}=x_{6}, \quad z_{6}=-x_{5} .
$$

For example, we give here the values of the parameters for $k+2=12$,

$$
\begin{aligned}
& v_{2}=-\sqrt{2}, \quad v_{3}=-\sqrt{\frac{1}{12}} . \\
& x_{1}=\sqrt{3}, \quad x_{5}=\frac{1}{6} . \\
& x_{4}=x_{6}=1 /(\sqrt{2} z)=\frac{\frac{1}{2}-\frac{i}{2}}{\sqrt{2}\left(-1+e^{\frac{i \pi}{3}}\right)\left(1+e^{\frac{i \pi}{3}}\right)\left(-1+e^{\frac{5 i \pi}{6}}\right)} . \\
& x_{2}=x_{3}=-r_{1}=-(\sqrt{3} / 2+1)^{1 / 2}+1 .
\end{aligned}
$$

To get the parameters for general $k$ we solved these equations for general $q=$ $\exp [\pi i /(k+2)]$ using symbolic manipulation, substituting some configurations. We find the following expressions for $v_{i}$.

$$
\begin{aligned}
& v_{1}=\frac{q^{11}+q^{9}+q^{7}}{q^{18}+q^{16}+2 q^{14}+3 q^{12}+3 q^{10}+3 q^{8}+3 q^{6}+2 q^{4}+q^{2}+1}, \\
& v_{2}=-\frac{q^{11}+q^{9}+q^{7}}{q^{18}+q^{16}+q^{14}+q^{12}+q^{10}+q^{8}+q^{6}+q^{4}+q^{2}+1}, \\
& v_{3}=-\frac{q^{6}\left(q^{12}+q^{10}+q^{8}+q^{6}+q^{4}+q^{2}+1\right)}{\left(q^{4}+1\right)\left(q^{6}+1\right)^{2}\left(q^{8}+q^{6}+q^{4}+q^{2}+1\right)} .
\end{aligned}
$$

For $x_{i}$ we find the following expressions,

$$
\begin{aligned}
& x_{1}=\frac{q^{10}+q^{6}}{q^{16}+q^{12}+q^{8}+q^{4}+1}, \\
& x_{2}=x_{3}=-\frac{q^{6}}{q^{12}+q^{8}+q^{6}+q^{4}+1}, \\
& x_{4}=x_{6}=\frac{q^{9}\left(q^{12}+q^{10}+q^{8}+q^{6}+q^{4}+q^{2}+1\right)}{\left(q^{2}+1\right)\left(q^{4}+1\right)\left(q^{12}+q^{8}+q^{6}+q^{4}+1\right)^{2}}, \\
& x_{5}=\frac{q^{6}\left(q^{12}+q^{10}+q^{8}+q^{6}+q^{4}+q^{2}+1\right)^{2}}{\left(q^{4}-q^{2}+1\right)^{2}\left(q^{6}+q^{4}+q^{2}+1\right)^{2}\left(q^{8}+q^{6}+q^{4}+q^{2}+1\right)^{2}} .
\end{aligned}
$$

The $z_{i}$ are given by eq. (6.32) from $x_{i}$. We find that the relations eqs. (6.28)-(6.30) are indeed obeyed for any $q$. 
Moreover, the following equations hold for the $\mathrm{SU}(2) 3 \times 3$ model:

$$
\begin{aligned}
K_{i} K_{i \pm 1} H_{i}= & H_{i \pm 1} K_{i} K_{i \pm 1}-\frac{q^{6}\left(q^{4}-1\right)\left(q^{14}-1\right)}{\left(q^{6}+1\right)^{2}\left(q^{8}-1\right)\left(q^{10}-1\right)}\left(K_{i} E_{i \pm 1}-E_{i} K_{i \pm 1}\right) \\
& -\frac{q^{7}\left(q^{4}-1\right)\left(q^{6}-1\right)}{\left(q^{6}+1\right)\left(q^{8}-1\right)\left(q^{10}-1\right)}\left(K_{i \pm 1} H_{i}-H_{i \pm 1} K_{i}\right) \\
& +\frac{q^{7}\left(q^{6}-1\right)}{q^{20}-1}\left(H_{i} K_{i \pm 1} H_{i}-H_{i \pm 1} K_{i} H_{i \pm 1}\right)
\end{aligned}
$$

and

$$
\begin{aligned}
K_{i+1} H_{i} K_{i+1}= & K_{i} H_{i+1} K_{i}+ \\
& +w_{1}\left(H_{i} K_{i+1}-K_{i} E_{i+1} K_{i}-K_{i} H_{i+1}+K_{i+1} E_{i} K_{i+1}+K_{i+1} H_{i}-H_{i+1} K_{i}\right) \\
& +w_{2}\left(K_{i+1} E_{i}-E_{i+1} K_{i}+H_{i}-H_{i+1}+E_{i} K_{i+1}-K_{i} E_{i+1}\right) \\
& +w_{3}\left(H_{i} K_{i+1} H_{i}-H_{i+1} K_{i} H_{i+1}\right)+w_{4}\left(E_{i}-E_{i+1}\right),
\end{aligned}
$$

where

$$
\begin{aligned}
& w_{1}=-\frac{\left(q^{4}-1\right)\left(q^{22}-1\right)}{q\left(q^{6}+1\right)\left(q^{8}-1\right)\left(q^{10}-1\right)}, \\
& w_{2}=\frac{\left(q^{4}-1\right)\left(q^{4}-q^{8}\right)\left(-q^{36}+q^{22}+q^{14}-1\right)}{q^{2}\left(q^{6}+1\right)^{2}\left(q^{8}-1\right)^{2}\left(q^{10}-1\right)^{2}}, \\
& w_{3}=\frac{q^{22}-1}{q\left(q^{20}-1\right)}, \\
& w_{4}=-\frac{\left(q^{4}-1\right)^{2}\left(q^{12}-1\right)\left(q^{14}-1\right)^{3}\left(q^{22}-1\right)}{q^{3}\left(q^{6}-1\right)\left(q^{6}+1\right)^{3}\left(q^{8}-1\right)^{3}\left(q^{10}-1\right)^{3}} .
\end{aligned}
$$

\section{$7 \quad H$ and $K$ relations for the general four blocks models}

We write $s_{i}=e^{\sqrt{-1} \zeta_{i}}, i \in\{0,1,2,3\}$. Let

$$
\begin{aligned}
G_{i} & =2^{3} s_{0}^{-\frac{3}{2}} \sin \left(\zeta_{0}\right) \sin \left(\zeta_{1}\right) \sin \left(\zeta_{2}\right) X_{i} \\
& =\frac{\sqrt{-1} P_{i}^{1}}{\sqrt{s_{0}} s_{1} s_{2}}-\frac{\sqrt{-1} P_{i}^{0}}{s_{0} \frac{5}{2} s_{1} s_{2}}+\frac{\sqrt{-1} s_{1} s_{2} P_{i}^{3}}{\sqrt{s_{0}}}-\frac{\sqrt{-1} s_{1} P_{i}^{2}}{\sqrt{s_{0}} s_{2}} .
\end{aligned}
$$

Then

$$
\begin{aligned}
G_{i}^{-1} & =-\frac{\sqrt{-1} \sqrt{s_{0}} P_{i}^{3}}{s_{1} s_{2}}-\sqrt{-1} \sqrt{s_{0}} s_{1} s_{2} P_{i}^{1}+\sqrt{-1} s_{0}^{\frac{5}{2}} s_{1} s_{2} P_{i}^{0}+\frac{\sqrt{-1} \sqrt{s_{0}} s_{2} P_{i}^{2}}{s_{1}}, \\
G_{i}^{2} & =-\frac{P_{i}^{1}}{s_{0} s_{1}^{2} s_{2}^{2}}-\frac{s_{1}^{2} P_{i}^{2}}{s_{0} s_{2}^{2}}-\frac{s_{1}^{2} s_{2}^{2} P_{i}^{3}}{s_{0}}-\frac{P_{i}^{0}}{s_{0}^{5} s_{1}^{2} s_{2}^{2}} .
\end{aligned}
$$

Let

$$
E_{i}=X_{i}\left(\zeta_{0}\right)=\frac{\left(s_{0}^{2} s_{1}^{2}-1\right)\left(s_{0}^{2} s_{2}^{2}-1\right)\left(s_{0}^{2}+1\right)}{\left(s_{1}^{2}-1\right)\left(s_{2}^{2}-1\right) s_{0}^{3}} P_{i}^{0} .
$$


The skein relation is $\sum_{a} P_{i}^{a}=1$. That is,

$$
\begin{aligned}
& G_{i}^{2}=\frac{E_{i}\left(s_{0}^{2} s_{1}^{2} s_{2}^{2}+1\right)\left(s_{1}^{2}-1\right)\left(s_{2}^{2}-1\right)}{\left(s_{0}^{2} s_{2}^{2}-1\right) s_{0}^{2} s_{1}^{2} s_{2}^{2}} \\
& +\frac{\sqrt{-1} G_{i}\left(s_{1}^{2} s_{2}^{2}-s_{1}^{2}+1\right)}{\sqrt{s_{0}} s_{1} s_{2}}-\frac{\left(s_{1}^{2} s_{2}^{2}-s_{2}^{2}+1\right)}{s_{0} s_{2}^{2}}+\frac{\sqrt{-1} s_{1} G_{i}^{-1}}{s_{0}^{\frac{3}{2}} s_{2}} .
\end{aligned}
$$

We also have

$$
\begin{aligned}
G_{i}^{-2}= & -\frac{s_{0}\left(s_{1}^{2} s_{2}^{2}-s_{1}^{2}+1\right)}{s_{1}^{2}}-\frac{\left(s_{0}^{2} s_{1}^{2} s_{2}^{2}+1\right)\left(s_{1}^{2}-1\right)\left(s_{2}^{2}-1\right) s_{0}^{2}}{\left(s_{0}^{2} s_{2}^{2}-1\right) s_{1}^{2}} E_{i} \\
& -\frac{\sqrt{-1} \sqrt{s_{0}}\left(s_{1}^{2} s_{2}^{2}-s_{2}^{2}+1\right)}{s_{1} s_{2}} G_{i}^{-1}-\frac{\sqrt{-1} s_{0}{ }^{\frac{3}{2}} s_{2}}{s_{1}} G_{i} .
\end{aligned}
$$

As in section 6 , we let $H_{i}=P_{i}^{3}=r_{1} 1_{i}+r_{2} E_{i}+r_{3} G_{i}+r_{4} G_{i}^{-1}$ and $K_{i}=r_{2} E_{i}+r_{1} 1_{i}+$ $r_{4} G_{i}+r_{3} G_{i}^{-1}$. Then

$$
\begin{aligned}
& r_{1}=\frac{\left(s_{1}^{2}-1\right) s_{2}^{2}}{\left(s_{1}^{2} s_{2}^{2}-1\right)\left(s_{2}^{2}+1\right)}, \\
& r_{2}=-\frac{\left(s_{1}^{2}-1\right)\left(s_{2}^{2}-1\right) s_{0} s_{2}^{2}}{\left(s_{0}^{2} s_{2}^{2}-1\right)\left(s_{1}^{2} s_{2}^{2}-1\right)\left(s_{2}^{2}+1\right)}, \\
& r_{3}=-\frac{\sqrt{-1} \sqrt{s_{0}} s_{1} s_{2}^{3}}{\left(s_{1}^{2} s_{2}^{2}-1\right)\left(s_{2}^{2}+1\right)}, \\
& r_{4}=-\frac{\sqrt{-1} s_{1} s_{2}}{\left(s_{1}^{2} s_{2}^{2}-1\right)\left(s_{2}^{2}+1\right) \sqrt{s_{0}}} .
\end{aligned}
$$

We express $G_{i}$ and $G_{i}^{-1}$ in terms of $1_{i}, E_{i}, H_{i}$, and $K_{i}$ and obtain

$$
\begin{aligned}
G_{i}= & \frac{E_{i}\left(r_{1} r_{4}-r_{2} r_{3}\right)}{\left(r_{3}^{2}-r_{4}^{2}\right)}-\frac{\left(r_{1} r_{3}-r_{2} r_{4}\right)}{\left(r_{3}^{2}-r_{4}^{2}\right)}+\frac{H_{i} r_{3}}{\left(r_{3}^{2}-r_{4}^{2}\right)}-\frac{K_{i} r_{4}}{\left(r_{3}^{2}-r_{4}^{2}\right)} \\
= & -\frac{\sqrt{-1} s_{0}^{\frac{3}{2}} s_{2}\left(s_{1}^{2}-1\right)}{\left(s_{0}^{2} s_{2}^{2}-1\right) s_{1}}+\frac{\sqrt{-1} E_{i} \sqrt{s_{0}} s_{2}\left(s_{1}^{2}-1\right)}{\left(s_{0}^{2} s_{2}^{2}-1\right) s_{1}} \\
& +\frac{\sqrt{-1} H_{i}\left(s_{1}^{2} s_{2}^{2}-1\right)\left(s_{2}^{2}+1\right) s_{0}^{\frac{3}{2}} s_{2}}{\left(s_{0}^{2} s_{2}^{4}-1\right) s_{1}}-\frac{\sqrt{-1} K_{i}\left(s_{1}^{2} s_{2}^{2}-1\right)\left(s_{2}^{2}+1\right) \sqrt{s_{0}}}{\left(s_{0}^{2} s_{2}^{4}-1\right) s_{1} s_{2}}, \\
G_{i}^{-1}= & \frac{\left(r_{1} r_{4}-r_{2} r_{3}\right)}{\left(r_{3}^{2}-r_{4}^{2}\right)}-\frac{E_{i}\left(r_{1} r_{3}-r_{2} r_{4}\right)}{\left(r_{3}^{2}-r_{4}^{2}\right)}-\frac{H_{i} r_{4}}{\left(r_{3}^{2}-r_{4}^{2}\right)}+\frac{K_{i} r_{3}}{\left(r_{3}^{2}-r_{4}^{2}\right)} \\
= & \frac{\sqrt{-1} \sqrt{s_{0}} s_{2}\left(s_{1}^{2}-1\right)}{\left(s_{0}^{2} s_{2}^{2}-1\right) s_{1}}-\frac{\sqrt{-1} E_{i} s_{0}{ }^{\frac{3}{2}} s_{2}\left(s_{1}^{2}-1\right)}{\left(s_{0}^{2} s_{2}^{2}-1\right) s_{1}} \\
& +\frac{\sqrt{-1} K_{i}\left(s_{1}^{2} s_{2}^{2}-1\right)\left(s_{2}^{2}+1\right) s_{0}^{\frac{3}{2}} s_{2}}{\left(s_{0}^{2} s_{2}^{4}-1\right) s_{1}}-\frac{\sqrt{-1} H_{i}\left(s_{1}^{2} s_{2}^{2}-1\right)\left(s_{2}^{2}+1\right) \sqrt{s_{0}}}{\left(s_{0}^{2} s_{2}{ }^{2}-1\right) s_{1} s_{2}} .
\end{aligned}
$$


We express $X_{i}(u)$ in terms of $1_{i}, E_{i}, H_{i}, K_{i}, e^{\sqrt{-1} u}$ and obtain $X_{i}(u)=p_{1} A+p_{2} A^{-1}+$ $p_{3} A^{-3}+p_{4} A^{3}$, where $A=e^{\sqrt{-1} u}$ and

$$
\begin{aligned}
& p_{1}=\frac{E_{i}\left(s_{1}^{2}-1\right) s_{0}\left(s_{0}^{2} s_{2}^{4}+s_{2}^{2}+1\right)}{\left(s_{0}^{2} s_{2}^{2}-1\right)}-\frac{\left(s_{1}^{2}-1\right)\left(s_{0}^{2} s_{2}^{4}+s_{0}^{2} s_{2}^{2}+1\right)}{\left(s_{0}^{2} s_{2}^{2}-1\right)} \\
& +\frac{H_{i}\left(s_{1}^{2} s_{2}^{2}-1\right)\left(s_{2}^{2}+1\right)\left(s_{0}^{2} s_{2}^{2}+s_{0}^{2}+1\right)}{\left(s_{0}^{2} s_{2}^{4}-1\right)} \\
& -\frac{K_{i}\left(s_{1}^{2} s_{2}^{2}-1\right)\left(s_{2}^{2}+1\right) s_{0}\left(s_{0}^{2} s_{2}^{2}+s_{2}^{2}+1\right)}{\left(s_{0}^{2} s_{2}^{4}-1\right)}, \\
& p_{2}=\frac{\left(s_{1}^{2}-1\right) s_{0}^{2}\left(s_{0}^{2} s_{2}^{4}+s_{2}^{2}+1\right)}{\left(s_{0}^{2} s_{2}^{2}-1\right)}-\frac{E_{i}\left(s_{1}^{2}-1\right) s_{0}\left(s_{0}^{2} s_{2}^{4}+s_{0}^{2} s_{2}^{2}+1\right)}{\left(s_{0}^{2} s_{2}^{2}-1\right)} \\
& -\frac{H_{i}\left(s_{1}^{2} s_{2}^{2}-1\right)\left(s_{2}^{2}+1\right) s_{0}^{2}\left(s_{0}^{2} s_{2}^{2}+s_{2}^{2}+1\right)}{\left(s_{0}^{2} s_{2}^{4}-1\right)} \\
& +\frac{K_{i}\left(s_{1}^{2} s_{2}^{2}-1\right)\left(s_{2}^{2}+1\right) s_{0}\left(s_{0}^{2} s_{2}^{2}+s_{0}^{2}+1\right)}{\left(s_{0}^{2} s_{2}^{4}-1\right)}, \\
& p_{3}=\frac{E_{i}\left(s_{1}^{2}-1\right) s_{0}^{3} s_{2}^{2}}{\left(s_{0}^{2} s_{2}^{2}-1\right)}-\frac{K_{i}\left(s_{1}^{2} s_{2}^{2}-1\right)\left(s_{2}^{2}+1\right) s_{0}^{3}}{\left(s_{0}^{2} s_{2}^{4}-1\right)} \\
& -\frac{\left(s_{1}^{2}-1\right) s_{0}{ }^{4} s_{2}^{2}}{\left(s_{0}^{2} s_{2}^{2}-1\right)}+\frac{H_{i}\left(s_{1}^{2} s_{2}^{2}-1\right)\left(s_{2}^{2}+1\right) s_{0}^{4} s_{2}^{2}}{\left(s_{0}^{2} s_{2}{ }^{4}-1\right)} \\
& p_{4}=\frac{\left(s_{1}^{2}-1\right) s_{2}^{2}}{\left(s_{0}^{2} s_{2}^{2}-1\right)}-\frac{H_{i}\left(s_{1}^{2} s_{2}^{2}-1\right)\left(s_{2}^{2}+1\right)}{\left(s_{0}^{2} s_{2}^{4}-1\right)} \\
& -\frac{E_{i}\left(s_{1}^{2}-1\right) s_{0} s_{2}^{2}}{\left(s_{0}^{2} s_{2}^{2}-1\right)}+\frac{K_{i}\left(s_{1}^{2} s_{2}^{2}-1\right)\left(s_{2}^{2}+1\right) s_{0} s_{2}^{2}}{\left(s_{0}^{2} s_{2}^{4}-1\right)} \text {. }
\end{aligned}
$$

Assume that $G_{i}, G_{i}^{-1}, E_{i}, 1_{i}$ satisfy the following relations (which are BMW relations and the new skein relation):

$$
\begin{aligned}
G_{i} G_{j} & =G_{j} G_{i}, \quad E_{i} G_{j}=G_{j} E_{i}, \quad|i-j| \geq 2, \\
G_{i} G_{j} G_{i} & =G_{j} G_{i} G_{j}, \quad|i-j|=1, \\
E_{i} E_{i \pm 1} E_{i} & =E_{i}, \\
G_{i} E_{i}=E_{i} G_{i} & =-\frac{\sqrt{-1}}{s_{0}^{\frac{5}{2}} s_{1} s_{2}} E_{i}, \\
E_{i} G_{j} E_{i} & =\sqrt{-1} s_{0}{ }^{\frac{5}{2}} s_{1} s_{2} E_{i}, \quad|i-j|=1, \\
E_{i}^{2} & =\frac{\left(s_{0}^{2} s_{1}^{2}-1\right)\left(s_{0}^{2} s_{2}^{2}-1\right)\left(s_{0}^{2}+1\right)}{\left(s_{1}^{2}-1\right)\left(s_{2}^{2}-1\right) s_{0}^{3}} E_{i} \\
E_{i} G_{i \pm 1} G_{i} & =G_{i \pm 1} G_{i} E_{i \pm 1}=E_{i} E_{i \pm 1}, \\
G_{i \pm 1} E_{i} E_{i \pm 1} & =G_{i}^{-1} E_{i \pm 1}, \\
E_{i} E_{i \pm 1} G_{i} & =E_{i} G_{i \pm 1}^{-1}, \\
G_{i \pm 1}^{-1} E_{i} G_{i \pm 1}^{-1} & =G_{i} E_{i \pm 1} G_{i},
\end{aligned}
$$


and

$$
\begin{aligned}
G_{i}^{2}= & \frac{E_{i}\left(s_{0}^{2} s_{1}^{2} s_{2}^{2}+1\right)\left(s_{1}^{2}-1\right)\left(s_{2}^{2}-1\right)}{\left(s_{0}^{2} s_{2}^{2}-1\right) s_{0}^{2} s_{1}^{2} s_{2}^{2}}+\frac{\sqrt{-1} G_{i}\left(s_{1}^{2} s_{2}^{2}-s_{1}^{2}+1\right)}{\sqrt{s_{0}} s_{1} s_{2}} \\
& -\frac{\left(s_{1}^{2} s_{2}^{2}-s_{2}^{2}+1\right)}{s_{0} s_{2}^{2}} 1_{i}+\frac{\sqrt{-1} s_{1} G_{i}^{-1}}{s_{0}^{\frac{3}{2}} s_{2}}, \\
G_{i}^{-2}= & -\frac{s_{0}\left(s_{1}^{2} s_{2}^{2}-s_{1}^{2}+1\right)}{s_{1}^{2}} 1_{i}-\frac{\left(s_{0}^{2} s_{1}^{2} s_{2}^{2}+1\right)\left(s_{1}^{2}-1\right)\left(s_{2}^{2}-1\right) s_{0}^{2}}{\left(s_{0}^{2} s_{2}^{2}-1\right) s_{1}^{2}} E_{i} \\
& -\frac{\sqrt{-1} \sqrt{s_{0}}\left(s_{1}^{2} s_{2}^{2}-s_{2}^{2}+1\right)}{s_{1} s_{2}} G_{i}^{-1}-\frac{\sqrt{-1} s_{0}{ }^{\frac{3}{2}} s_{2}}{s_{1}} G_{i} .
\end{aligned}
$$

By expression $H_{i}, K_{i}$ in terms of $1_{i}, G_{i}, G_{i}^{-1}, E_{i}$ and use the above relations, it is easy to check that the following relations hold.

$$
\begin{aligned}
& E_{i}^{2}=\frac{\left(s_{0}^{2} s_{1}^{2}-1\right)\left(s_{0}^{2} s_{2}^{2}-1\right)\left(s_{0}^{2}+1\right)}{\left(s_{1}^{2}-1\right)\left(s_{2}^{2}-1\right) s_{0}^{3}} E_{i}, \\
& H_{i}^{2}=H_{i}, \\
& K_{i}^{2}=v_{1} K_{i}+v_{2} H_{i}+v_{3} 1_{i}+v_{4} E_{i}, \\
& H_{i} E_{i}=E_{i} H_{i}=0 \text {, } \\
& K_{i} E_{i}=E_{i} K_{i}=q_{1} E_{i}, \\
& K_{i} H_{i}=H_{i} K_{i}=q_{2} H_{i}, \\
& E_{i} K_{i \pm 1} E_{i}=0, \\
& E_{i} E_{i \pm 1} H_{i}=E_{i} K_{i \pm 1}, \\
& E_{i} E_{i \pm 1} K_{i}=E_{i} H_{i \pm 1} \\
& H_{i} E_{i \pm 1} E_{i}=K_{i \pm 1} E_{i}, \\
& K_{i} E_{i \pm 1} E_{i}=H_{i \pm 1} E_{i}, \\
& E_{i} H_{i \pm 1} E_{i}=q_{1} E_{i} \\
& E_{i} H_{i \pm 1} H_{i}=q_{2} E_{i} K_{i \pm 1}=E_{i} K_{i \pm 1} K_{i}, \\
& H_{i} H_{i \pm 1} E_{i}=q_{2} K_{i \pm 1} E_{i}=K_{i} K_{i \pm 1} E_{i} \text {, } \\
& K_{i} E_{i \pm 1} H_{i}=H_{i \pm 1} E_{i} K_{i \pm 1}, \\
& H_{i} E_{i \pm 1} H_{i}=K_{i \pm 1} E_{i} K_{i \pm 1} \text {, } \\
& E_{i} H_{i+1} K_{i}-E_{i+1} H_{i} K_{i+1}=E_{i}\left(v_{1} H_{i+1}+v_{2} K_{i+1}+v_{3} E_{i+1}+v_{4} 1_{i}\right) \\
& +E_{i+1}\left(-v_{1} H_{i}-v_{2} K_{i}-v_{3} E_{i}-v_{4} 1_{i}\right), \\
& K_{i} H_{i+1} E_{i}-K_{i+1} H_{i} E_{i+1}=\left(v_{1} H_{i+1}+v_{2} K_{i+1}+v_{3} E_{i+1}+v_{4} 1_{i}\right) E_{i} \\
& +\left(-v_{1} H_{i}-v_{2} K_{i}-v_{3} E_{i}-v_{4} 1_{i}\right) E_{i+1}, \\
& H_{i} K_{i \pm 1} E_{i}-E_{i \pm 1} K_{i} H_{i \pm 1}=K_{i \pm 1} E_{i}-E_{i \pm 1} K_{i}, \\
& E_{i} H_{i \pm 1} K_{i}-K_{i} H_{i \pm 1} E_{i}=E_{i}\left(v_{1} H_{i \pm 1}+v_{2} K_{i \pm 1}+v_{3} E_{i \pm 1}\right) \\
& -\left(v_{1} H_{i \pm 1}+v_{2} K_{i \pm 1}+v_{3} E_{i \pm 1}\right) E_{i} .
\end{aligned}
$$


where

$$
\begin{aligned}
& q_{1}=-\frac{\left(s_{0}^{2} s_{1}^{2} s_{2}^{2}-1\right)\left(-s_{0}{ }^{6} s_{2}{ }^{6}+s_{0}{ }^{4} s_{2}{ }^{2}+s_{0}{ }^{2} s_{2}{ }^{4}-1\right)}{\left(s_{0}^{2} s_{2}^{2}-1\right)\left(s_{1}^{2} s_{2}^{2}-1\right)\left(s_{2}^{4}-1\right) s_{0}{ }^{3}}, \\
& q_{2}=\frac{\left(s_{1}^{2}-s_{0}^{2} s_{2}^{2}\right)\left(s_{0}^{2}-1\right) s_{2}^{2}}{\left(s_{0}^{2} s_{2}^{2}-1\right)\left(s_{1}^{2} s_{2}^{2}-1\right)\left(s_{2}^{2}+1\right) s_{0}}, \\
& v_{1}=\frac{\left(s_{1}^{2}-1\right)\left(-s_{0}{ }^{4} s_{2}{ }^{6}-s_{0}{ }^{2} s_{2}{ }^{4}+s_{0}{ }^{2} s_{2}{ }^{2}+1\right)}{\left(s_{0}^{2} s_{2}^{2}-1\right)\left(s_{1}^{2} s_{2}^{2}-1\right)\left(s_{2}^{2}+1\right) s_{0}} \text {, } \\
& v_{2}=\frac{\left(s_{0}^{2} s_{2}^{2}+1\right)\left(s_{1}^{2}-s_{0}^{2} s_{2}^{2}\right)}{\left(s_{1}^{2} s_{2}^{2}-1\right)\left(s_{2}^{2}+1\right) s_{0}^{2}}, \\
& v_{3}=\frac{\left(s_{0}^{2} s_{2}{ }^{4}-1\right)^{2}\left(s_{0}^{4} s_{1}^{2} s_{2}{ }^{4}-s_{0}{ }^{2} s_{1}{ }^{4} s_{2}{ }^{2}-s_{0}{ }^{2} s_{2}{ }^{2}+s_{1}{ }^{2}\right)}{\left(s_{0}^{2} s_{2}^{2}-1\right)^{2}\left(s_{1}^{2} s_{2}^{2}-1\right)^{2}\left(s_{2}^{2}+1\right)^{2} s_{0}^{2}}, \\
& v_{4}=\frac{\left(s_{0}^{2} s_{2}^{2}+1\right)\left(s_{0}^{2} s_{2}^{4}-1\right)^{2}\left(s_{0}^{2} s_{1}^{2} s_{2}^{2}-1\right)\left(s_{1}^{2}-1\right)}{\left(s_{0}^{2} s_{2}^{2}-1\right)\left(s_{1}^{2} s_{2}^{2}-1\right)^{2}\left(s_{2}^{2}-1\right)\left(s_{2}^{2}+1\right)^{2} s_{0}^{3}} \text {. }
\end{aligned}
$$

The equations in (7.3) follow from the equations in (7.1) and (7.2).

We expand the Yang-Baxter equation

$$
X_{i}(u) X_{i+1}(u+v) X_{i}(v)=X_{i+1}(v) X_{i}(u+v) X_{i+1}(u)
$$

using $X_{i}(u)=p_{1} A+p_{2} A^{-1}+p_{3} A^{-3}+p_{4} A^{3}$ and obtain 37 equations in terms of $1_{i}, H_{i}$, $K_{i}, E_{i}$. Assume that $H_{i}, K_{i}, E_{i}$ satisfy (7.1), (7.2), (7.3). We simplify the 37 equations using (7.1), (7.2), (7.3) and obtain 37 equations which involve terms of the form $H_{i} H_{i+1} H_{i}$, $K_{i} K_{i+1} H_{i}, K_{i} K_{i+1} K_{i}, K_{i} H_{i+1} H_{i}, K_{i+1} H_{i} K_{i+1}$. We combine the 37 equations and solve the equations for $H_{i} H_{i+1} H_{i}, K_{i} K_{i+1} H_{i}, K_{i+1} K_{i} K_{i+1}, K_{i} H_{i+1} H_{i}, K_{i+1} H_{i} K_{i+1}$ and obtain the following:

$$
\begin{aligned}
H_{i} H_{i+1} H_{i}= & H_{i+1} H_{i} H_{i+1}+a_{1}\left(H_{i}-H_{i+1}\right) \\
& +a_{2}\left(K_{i} E_{i+1} K_{i}-K_{i+1} E_{i} K_{i+1}\right)+a_{3}\left(H_{i} K_{i+1} H_{i}-H_{i+1} K_{i} H_{i+1}\right), \\
K_{i} K_{i \pm 1} H_{i}= & H_{i \pm 1} K_{i} K_{i \pm 1}+a_{1}\left(E_{i} K_{i \pm 1}-K_{i} E_{i \pm 1}\right) \\
& +a_{2}\left(H_{i \pm 1} K_{i}-K_{i \pm 1} H_{i}\right)+a_{3}\left(H_{i} K_{i \pm 1} H_{i}-H_{i \pm 1} K_{i} H_{i \pm 1}\right), \\
K_{i+1} K_{i} K_{i+1}= & K_{i} K_{i+1} K_{i}+b_{1}\left(E_{i} K_{i+1}-K_{i} E_{i+1}+K_{i+1} E_{i}-E_{i+1} K_{i}\right) \\
& +b_{2}\left(-H_{i+1} K_{i}+K_{i+1} H_{i}+H_{i} K_{i+1}-K_{i} H_{i+1}\right) \\
& +b_{3}\left(H_{i} K_{i+1} H_{i}-H_{i+1} K_{i} H_{i+1}\right)+b_{4}\left(-K_{i}+K_{i+1}\right), \\
K_{i} H_{i \pm 1} H_{i}= & H_{i \pm 1} H_{i} K_{i \pm 1} \\
& +b_{1}\left(K_{i} E_{i \pm 1}-E_{i} K_{i \pm 1}-H_{i}+H_{i \pm 1}\right) \\
& +b_{2}\left(-K_{i \pm 1} H_{i}+H_{i \pm 1} K_{i}+K_{i} E_{i \pm 1} K_{i}-K_{i \pm 1} E_{i} K_{i \pm 1}\right) \\
& +b_{3}\left(-H_{i} K_{i \pm 1} H_{i}+H_{i \pm 1} K_{i} H_{i \pm 1}\right) \\
& +b_{4}\left(H_{i \pm 1} E_{i}-E_{i \pm 1} H_{i}\right),
\end{aligned}
$$




$$
\begin{aligned}
K_{i+1} H_{i} K_{i+1}= & K_{i} H_{i+1} K_{i} \\
& +c_{1}\left(-H_{i} K_{i+1}+K_{i} E_{i+1} K_{i}+K_{i} H_{i+1}-K_{i+1} E_{i} K_{i+1}-K_{i+1} H_{i}+H_{i+1} K_{i}\right) \\
& +c_{2}\left(-K_{i+1} E_{i}+E_{i+1} K_{i}-H_{i}+H_{i+1}-E_{i} K_{i+1}+K_{i} E_{i+1}\right) \\
& +c_{3}\left(H_{i} K_{i+1} H_{i}-H_{i+1} K_{i} H_{i+1}\right)+c_{4}\left(-E_{i}+E_{i+1}\right)
\end{aligned}
$$

where

$$
\begin{aligned}
& a_{1}=\frac{\left(s_{0}^{2} s_{2}^{4}-1\right)\left(s_{1}^{2}-1\right) s_{2}^{2}}{\left(s_{0}^{2} s_{2}^{2}-1\right)\left(s_{1}^{2} s_{2}^{2}-1\right)\left(s_{2}^{2}+1\right)^{2}}, \\
& a_{2}=\frac{\left(s_{1}^{2}-1\right)\left(s_{2}^{2}-1\right) s_{0} s_{2}^{2}}{\left(s_{0}^{2} s_{2}^{2}-1\right)\left(s_{1}^{2} s_{2}^{2}-1\right)\left(s_{2}^{2}+1\right)}, \\
& a_{3}=\frac{\left(s_{2}^{2}-1\right) s_{0} s_{2}^{2}}{s_{0}^{2} s_{2}{ }^{6}-1}, \\
& b_{1}=-\frac{\left(s_{0}^{2} s_{2}^{4}-1\right)\left(s_{1}^{2}-s_{0}^{2} s_{2}^{2}\right)\left(s_{1}^{2}-1\right) s_{2}^{2}}{\left(s_{0}^{2} s_{2}^{2}-1\right)\left(s_{1}^{2} s_{2}^{2}-1\right)^{2}\left(s_{2}^{2}+1\right)^{2} s_{0}}, \\
& b_{2}=-\frac{\left(s_{1}^{2}-1\right) s_{2}^{2}}{\left(s_{1}^{2} s_{2}^{2}-1\right)\left(s_{2}^{2}+1\right)} \text {, } \\
& b_{3}=-\frac{\left(s_{2}^{2}-s_{0}^{2} s_{2}^{4}\right)}{\left(s_{0}^{2} s_{2}{ }^{6}-1\right)}, \\
& b_{4}=\frac{\left(s_{0}^{2} s_{2}^{4}-1\right)^{2}\left(s_{1}^{2}-1\right)^{2} s_{2}^{2}}{\left(s_{0}^{2} s_{2}^{2}-1\right)^{2}\left(s_{1}^{2} s_{2}^{2}-1\right)^{2}\left(s_{2}^{2}+1\right)^{2}}, \\
& c_{1}=\frac{\left(s_{0}^{4} s_{2}{ }^{6}-1\right)\left(s_{1}^{2}-1\right)}{\left(s_{0}^{2} s_{2}^{2}-1\right)\left(s_{1}^{2} s_{2}^{2}-1\right)\left(s_{2}^{2}+1\right) s_{0}}, \\
& c_{2}=-\frac{\left(s_{1}{ }^{2}-s_{0}{ }^{2} s_{2}{ }^{2}\right)\left(s_{1}{ }^{2}-1\right)\left(-s_{0}{ }^{6} s_{2}{ }^{10}+s_{0}{ }^{4} s_{2}{ }^{6}+s_{0}{ }^{2} s_{2}{ }^{4}-1\right)}{\left(s_{0}{ }^{2} s_{2}{ }^{2}-1\right)^{2}\left(s_{1}{ }^{2} s_{2}{ }^{2}-1\right)^{2}\left(s_{2}{ }^{2}+1\right)^{2} s_{0}{ }^{2}}, \\
& c_{3}=\frac{s_{0}{ }^{4} s_{2}{ }^{6}-1}{s_{0}{ }^{3} s_{2}{ }^{6}-s_{0}}, \\
& c_{4}=\frac{\left(s_{0}{ }^{2} s_{2}{ }^{4}-1\right)^{3}\left(s_{0}{ }^{4} s_{2}{ }^{6}-1\right)\left(s_{0}{ }^{2} s_{1}{ }^{2} s_{2}{ }^{2}-1\right)\left(s_{1}{ }^{2}-1\right)^{2}}{\left(s_{0}^{2} s_{2}{ }^{2}-1\right)^{3}\left(s_{1}^{2} s_{2}{ }^{2}-1\right)^{3}\left(s_{2}{ }^{2}-1\right)\left(s_{2}{ }^{2}+1\right)^{3} s_{0}{ }^{3}} .
\end{aligned}
$$

On the other hand, we verified that the equations (7.1)-(7.8) imply the Yang-Baxter equation

$$
X_{i}(u) X_{i+1}(u+v) X_{i}(v)=X_{i+1}(v) X_{i}(u+v) X_{i+1}(u) .
$$

Let $\mathbb{K}$ be a field. We define a $\mathbb{K}\left(s_{0}, s_{1}, s_{2}\right)$-algebra $A$ generated by $H_{i}, K_{i}, E_{i}, i \in$ $\{1, \ldots, n\}$ subject to the relations (7.1)-(7.8). We have the following theorem.

Suppose that $H_{i}, K_{i}, E_{i}, i \in\{1, \ldots, n\}$ satisfy (7.1)-(7.3). Then $X_{i}(u)$ satisfies YBE if and only if $H_{i}, K_{i}, E_{i}$ satisfy the other defining relations in the algebra $A$.

When $s_{0}=q, s_{1}=q^{2}, s_{2}=q^{3}$ (SU(2) fused $3 \times 3$ lattice model case), the equations (7.4)-(7.8) are (6.28), (6.44), (6.30), (6.29), and (6.45) respectively. When $s_{0}=q^{-6}$, $s_{1}=q^{-1}, s_{2}=q^{4}\left(G_{2}\right.$ case), the relations (7.1)-(7.8) are the corresponding relations in section 3 of ref. [9] after some rescaling of $E_{i}, H_{i}, K_{i}$. 
Since we showed in section 3 that the YBE and BMW are obeyed if and only if the relation $g$, eqs. (3.19)-(3.21), is obeyed, it follows that this relation is equivalent to the algebra $A$ or to the general $H$ and $K$ relations.

\section{Conclusions}

We studied in this paper the algebraic structure underlining solvable IRF (Interaction Round the Face) lattice models. We proposed that the algebra depends only on the number of blocks. For two blocks we obtain the Hecke $A_{N}$ algebra and the Temperley-Lieb (TL) algebra. For three blocks, we found that it is the weak Birman-Murakami-Wenzl (BMW) algebra. This algebra has a quotient which is the BMW algebra, which contains the TL algebra, with a different skein relation. In fact, in all cases we checked the all the BMW relations hold. We conjecture that this phenomenon is general. For four blocks, we found that the algebra is a version of BMW algebra, with a different skein relation, along with one additional relation. This algebra is new, to the best of our knowledge, and we plan to further investigate it.

These results suggest the following general picture. The $n$ block algebras, for $n=$ $1,2, \ldots$ form a chain of algebras, which are quotients of the universal free algebra with generators $1, E_{i}, G_{i}$, such that the relations which define the $n+1$ th quotient contain those of the $n$th quotient, except for the skein relation which is different. We find that this picture is established for the two, three and four blocks cases.

To further investigate this chain of algebras, it behooves us to study the five blocks algebra, and hopefully also higher blocks, with the ultimate goal of finding the general algebra. This we intend to pursue in future work.

If the conjecture of section 4 holds, then our results will be relevant to knot theory, since then the algebras can be used to define new link invariants. In addition, this algebraic structure we found sheds more light into the physics of IRF models.

\section{Acknowledgments}

We thank Hans Wenzl for very helpful discussions. D.G. thank the theory department of CERN for the hospitality while part of this work was done. We thank Ida Deichaite for remarks on the manuscript. R.T. was supported by a research grant from the center for new scientists, Weizmann Institute of Science, and by the Israel Science Foundation (grant No. 335/19). J.L. was supported by the Austrian Science Fund (FWF): M 2633-N32 Meitner Program. The research of V.B. was supported by the RFBR under grant 18-02-01131. R.T. is an incumbent of the Lilian and George Lyttle career development chair.

\section{A Weights of the $G_{2}$ model}

The Boltzmann weights are taken from Kuniba and Suzuki, ref. [16]. 
The Weyl vector $\rho=(1,1)$ and the weights of the seven-dimensional representation space of $U_{q}\left(G_{2}\right)$ are

$$
\begin{aligned}
& e_{-3}=(0,1), \quad e_{-2}=(1,-1), \\
& e_{-1}=(-1,2), \quad e_{0}=(0,0),
\end{aligned}
$$

and

$$
e_{i}=-e_{-i} .
$$

We consider the highest weight module of $U_{q}\left(G_{2}\right)$ with the highest weight

$$
a=\left(a_{1}, a_{2}\right)
$$

We introduce function

$$
G[a]=s\left[3 a_{1}\right] s\left[a_{2}\right] s\left[3 a_{1}+a_{2}\right] s\left[3 a_{1}+2 a_{2}\right] s\left[3 a_{1}+3 a_{2}\right] s\left[6 a_{1}+3 a_{2}\right] .
$$

Now we introduce the Boltzmann weights. First of all, for $\mu \neq 0$

$$
\begin{aligned}
& B[a, u, \mu, \mu, \mu, \mu]=\frac{s[1+u] s[4+u] s[6+u]}{s[1] s[4] s[6]} \\
& B[a, u,-3,-2,-3,-2]=\frac{s\left[a_{2}-u\right] s[4+u] s[6+u]}{s\left[a_{2}\right] s[4] s[6]} \\
& B[a, u,-2,-3,-3,-2]=\left(\frac{s\left[a_{2}+1\right] s\left[a_{2}-1\right]}{s\left[a_{2}\right]^{2}}\right)^{\frac{1}{2}} \frac{s[u] s[4+u] s[6+u]}{s[1] s[4] s[6]} \\
& B[a, u,-3,-1,-3,-1]=\frac{s\left[3 a_{1}+a_{2}-u\right] s[4+u] s[6+u]}{s\left[3 a_{1}+a_{2}\right] s[4] s[6]} \\
& B[a, u,-1,-3,-3,-1]=\left(\frac{s\left[3 a_{1}+a_{2}+1\right] s\left[3 a_{1}+a_{2}-1\right]}{s\left[3 a_{1}+a_{2}\right]^{2}}\right)^{\frac{1}{2}} \frac{s[u] s[4+u] s[6+u]}{s[1] s[4] s[6]} \\
& B[a, u,-2,1,-2,1]=\frac{s\left[3 a_{1}+2 a_{2}-u\right] s[4+u] s[6+u]}{s\left[3 a_{1}+2 a_{2}\right] s[4] s[6]} \\
& B[a, u, 1,-2,-2,1]=\left(\frac{s\left[3 a_{1}+2 a_{2}+1\right] s\left[3 a_{1}+2 a_{2}-1\right]}{s\left[3 a_{1}+2 a_{2}\right]^{2}}\right)^{\frac{1}{2}} \frac{s[u] s[4+u] s[6+u]}{s[1] s[4] s[6]} \\
& B[a, u,-3,0,-3,0]=\frac{s\left[3 a_{1}+a_{2}-2-u\right] s\left[a_{2}-u\right] s[6+u]}{s\left[3 a_{1}+a_{2}-2\right] s\left[a_{2}\right] s[6]}+ \\
& +\frac{s\left[a_{2}+3\right] s\left[3 a_{1}+a_{2}-1\right] s\left[3 a_{1}+a_{2}+2\right] s\left[3 a_{1}+2 a_{2}-2-u\right] s[2] s[u] s[6+u]}{s\left[a_{2}\right] s\left[3 a_{1}+a_{2}\right] s\left[3 a_{1}+a_{2}-2\right] s\left[3 a_{1}+2 a_{2}+1\right] s[1] s[4] s[6]} \\
& B[a, u, 0,-3,-3,0]=\frac{s[u] s[3+u] s[6+u]}{s[1] s[4] s[6]} \times \\
& \times\left(\frac{s\left[a_{2}-1\right] s\left[a_{2}+2\right] s\left[3 a_{1}+a_{2}-1\right] s\left[3 a_{1}+a_{2}+2\right] s\left[3 a_{1}+2 a_{2}-1\right] s\left[3 a_{1}+2 a_{2}+3\right]}{s\left[a_{2}\right] s\left[a_{2}+1\right] s\left[3 a_{1}+a_{2}\right] s\left[3 a_{1}+a_{2}+1\right] s\left[3 a_{1}+2 a_{2}+1\right]^{2}}\right)^{\frac{1}{2}} \\
& B[a, u,-2,-1,0,-3]=\frac{s\left[a_{2}+3+u\right] s[u] s[6+u]}{s\left[3 a_{1}+a_{2}+1\right] s[4] s[6]} \times \\
& \times\left(\frac{s\left[3 a_{1}+3\right] s\left[3 a_{1}+a_{2}-1\right] s\left[3 a_{1}+a_{2}+2\right] s\left[3 a_{1}+2 a_{2}-1\right] s[2]}{s\left[3 a_{1}\right] s\left[3 a_{1}+2 a_{2}+1\right] s\left[a_{2}\right] s\left[a_{2}+1\right] s[1]}\right)^{\frac{1}{2}}
\end{aligned}
$$




$$
\begin{aligned}
& B[a, u,-1,-2,0,-3]=-\frac{s\left[3 a_{1}+a_{2}+3+u\right] s[u] s[6+u]}{s\left[a_{2}+1\right] s[4] s[6]} \times \\
& \times\left(\frac{s\left[3 a_{1}-3\right] s\left[a_{2}-1\right] s\left[a_{2}+2\right] s\left[3 a_{1}+2 a_{2}-1\right] s[2]}{s\left[3 a_{1}\right] s\left[3 a_{1}+2 a_{2}+1\right] s\left[3 a_{1}+a_{2}\right] s\left[3 a_{1}+a_{2}+1\right] s[1]}\right)^{\frac{1}{2}} \\
& B[a, u,-2,-1,-2,-1]= \\
& =\frac{s\left[3 a_{1}-u\right] s[2+u] s[6+u]}{s\left[3 a_{1}\right] s[2] s[6]}+\frac{s\left[a_{2}-2\right] s\left[3 a_{1}+a_{2}+3\right] s\left[3 a_{1}-2-u\right] s[u] s[6+u]}{s\left[a_{2}\right] s\left[3 a_{1}+a_{2}+1\right] s\left[3 a_{1}\right] s[4] s[6]} \\
& B[a, u,-3,1,-3,1]=\frac{s\left[3 a_{1}+3 a_{2}-u\right] s[2+u] s[6+u]}{s\left[3 a_{1}+3 a_{2}\right] s[2] s[6]}+ \\
& +\frac{s\left[a_{2}+2\right] s\left[3 a_{1}+2 a_{2}+3\right] s\left[3 a_{1}+3 a_{2}-2-u\right] s[u] s[6+u]}{s\left[a_{2}\right] s\left[3 a_{1}+2 a_{2}+1\right] s\left[3 a_{1}+3 a_{2}\right] s[4] s[6]} \\
& B[a, u, 1,-3,0,-2]=-\frac{s\left[3 a_{1}+2 a_{2}+3+u\right] s[u] s[6+u]}{s\left[a_{2}-1\right] s[4] s[6]} \times \\
& \times\left(\frac{s\left[a_{2}-2\right] s\left[a_{2}+1\right] s\left[3 a_{1}+a_{2}-1\right] s\left[3 a_{1}+3 a_{2}-3\right] s[2]}{s\left[3 a_{1}+a_{2}+1\right] s\left[3 a_{1}+3 a_{2}\right] s\left[3 a_{1}+2 a_{2}\right] s\left[3 a_{1}+2 a_{2}+1\right] s[1]}\right)^{\frac{1}{2}} \\
& B[a, u,-2,0,-2,0]=\frac{s\left[3 a_{1}+2 a_{2}-2-u\right] s\left[a_{2}+u\right] s[6+u]}{s\left[3 a_{1}+2 a_{2}-2\right] s\left[a_{2}\right] s[6]}+ \\
& +\frac{s\left[a_{2}-3\right] s\left[3 a_{1}+2 a_{2}-1\right] s\left[3 a_{1}+2 a_{2}+2\right] s\left[3 a_{1}+a_{2}-2-u\right] s[2] s[u] s[6+u]}{s\left[a_{2}\right] s\left[3 a_{1}+2 a_{2}\right] s\left[3 a_{1}+2 a_{2}-2\right] s\left[3 a_{1}+a_{2}+1\right] s[1] s[4] s[6]} \\
& B[a, u, 0,-2,-2,0]=\frac{s[u] s[3+u] s[6+u]}{s[1] s[4] s[6]} \times \\
& \times\left(\frac{s\left[a_{2}-2\right] s\left[a_{2}+1\right] s\left[3 a_{1}+a_{2}-1\right] s\left[3 a_{1}+a_{2}+3\right] s\left[3 a_{1}+2 a_{2}-1\right] s\left[3 a_{1}+2 a_{2}+2\right]}{s\left[a_{2}-1\right] s\left[a_{2}\right] s\left[3 a_{1}+a_{2}+1\right]^{2} s\left[3 a_{1}+2 a_{2}+1\right] s\left[3 a_{1}+2 a_{2}\right]}\right)^{\frac{1}{2}} \\
& B[a, u,-3,2,-3,2]=\frac{s\left[6 a_{1}+3 a_{2}-u\right] s[2+u] s[6+u]}{s\left[6 a_{1}+3 a_{2}\right] s[2] s[6]}+ \\
& +\frac{s\left[3 a_{1}+a_{2}+2\right] s\left[3 a_{1}+2 a_{2}+3\right] s\left[6 a_{1}+3 a_{2}-2-u\right] s[u] s[6+u]}{s\left[3 a_{1}+a_{2}\right] s\left[3 a_{1}+2 a_{2}+1\right] s\left[6 a_{1}+3 a_{2}\right] s[4] s[6]} \\
& B[a, u,-1,0,-1,0]=\frac{s\left[3 a_{1}+a_{2}+u\right] s\left[3 a_{1}+2 a_{2}-2-u\right] s[6+u]}{s\left[3 a_{1}+a_{2}\right] s\left[3 a_{1}+2 a_{2}-2\right] s[6]}+ \\
& +\frac{s\left[3 a_{1}+a_{2}-3\right] s\left[3 a_{1}+2 a_{2}-1\right] s\left[3 a_{1}+2 a_{2}+2\right] s\left[a_{2}-2-u\right] s[2] s[u] s[6+u]}{s\left[3 a_{1}+a_{2}\right] s\left[3 a_{1}+2 a_{2}-2\right] s\left[3 a_{1}+2 a_{2}\right] s\left[a_{2}+1\right] s[1] s[4] s[6]} \\
& B[a, u, 0,-1,-1,0]=\frac{s[u] s[3+u] s[6+u]}{s[1] s[4] s[6]} \\
& \times\left(\frac{s\left[a_{2}-1\right] s\left[a_{2}+3\right] s\left[3 a_{1}+a_{2}-2\right] s\left[3 a_{1}+a_{2}+1\right] s\left[3 a_{1}+2 a_{2}-1\right] s\left[3 a_{1}+2 a_{2}+2\right]}{s\left[a_{2}+1\right]^{2} s\left[3 a_{1}+a_{2}-1\right] s\left[3 a_{1}+a_{2}\right] s\left[3 a_{1}+2 a_{2}\right] s\left[3 a_{1}+2 a_{2}+1\right]}\right)^{\frac{1}{2}} \\
& B[a, u, 3,-3,3,-3]=\frac{s\left[6 a_{1}+4 a_{2}-1+u\right] s[2+u] s[6+u]}{s\left[6 a_{1}+4 a_{2}-1\right] s[2] s[6]} \\
& -\frac{s\left[a_{2}-2\right] s\left[3 a_{1}+a_{2}-2\right] s\left[3 a_{1}+2 a_{2}-3\right] s\left[6 a_{1}+4 a_{2}+1+u\right] s[u] s[6+u]}{s\left[a_{2}\right] s\left[3 a_{1}+a_{2}\right] s\left[3 a_{1}+2 a_{2}-1\right] s\left[6 a_{1}+4 a_{2}-1\right] s[4] s[6]} \\
& -\frac{G\left[a+e_{3}\right]}{G[a]} \frac{s\left[6 a_{1}+4 a_{2}+5+u\right] s[u] s[2+u]}{s\left[6 a_{1}+4 a_{2}-1\right] s[4] s[6]}
\end{aligned}
$$




$$
\begin{aligned}
& B[a, u, 2,-2,2,-2]=\frac{s\left[6 a_{1}+2 a_{2}-1+u\right] s[2+u] s[6+u]}{s\left[6 a_{1}+2 a_{2}-1\right] s[2] s[6]} \\
& -\frac{s\left[a_{2}+2\right] s\left[3 a_{1}+a_{2}-3\right] s\left[3 a_{1}+2 a_{2}-2\right] s\left[6 a_{1}+2 a_{2}+1+u\right] s[u] s[6+u]}{s\left[a_{2}\right] s\left[3 a_{1}+a_{2}-1\right] s\left[3 a_{1}+2 a_{2}\right] s\left[6 a_{1}+2 a_{2}-1\right] s[4] s[6]} \\
& -\frac{G\left[a+e_{2}\right]}{G[6]} \frac{s\left[6 a_{1}+2 a_{2}+5+u\right] s[u] s[2+u]}{s\left[6 a_{1}+2 a_{2}-1\right] s[4] s[6]} \\
& B[a, u, 1,-1,1,-1]= \\
& =\frac{s\left[2 a_{2}-1+u\right] s[2+u] s[6+u]}{s\left[2 a_{2}-1\right] s[2] s[6]} \\
& -\frac{s\left[a_{2}-3\right] s\left[3 a_{1}+a_{2}+2\right] s\left[3 a_{1}+2 a_{2}-2\right] s\left[2 a_{2}+1+u\right] s[u] s[6+u]}{s\left[a_{2}-1\right] s\left[3 a_{1}+a_{2}\right] s\left[3 a_{1}+2 a_{2}\right] s\left[2 a_{2}-1\right] s[4] s[6]} \\
& -\frac{G\left[a+e_{1}\right]}{G\left[2 a_{2}+5+u\right] s[u] s[2+u]} \\
& s\left[2 a_{2}-1\right] s[4] s[6] \\
& B[a, u, 0,0,0,0]=\frac{s[6-u] s[12+u] s[3+u]}{s[6] s[12] s[3]}+\frac{s[u] s[3+u] s[6+u]}{s[6] s[9] s[12]} \times \\
& \quad \times \sum_{\mu=1}^{3}\left(\frac{G\left[a+e_{\mu}\right]}{G[a]} B[a,-12,0, \mu, 0, \mu]+\frac{G\left[a+e_{-\mu}\right]}{G[a]} B[a,-12,0,-\mu, 0,-\mu]\right)
\end{aligned}
$$

Taking symmetries (eq. (9a) in ref. [16]) into account, we get also

$$
\begin{aligned}
B[a, u,-3,-2,-2,-3] & =B[a, u,-2,-3,-3,-2] \\
B[a, u,-3,-1,-1,-3] & =B[a, u,-1,-3,-3,-1] \\
B[a, u,-2,1,1,-2] & =B[a, u, 1,-2,-2,1] \\
B[a, u,-3,0,0,-3] & =B[a, u, 0,-3,-3,0] \\
B[a, u, 0,-3,-2,-1] & =B[a, u,-2,-1,0,-3] \\
B[a, u, 0,-3,-1,-2] & =B[a, u,-1,-2,0,-3] \\
B[a, u, 0,-2,1,-3] & =B[a, u, 1,-3,0,-2] \\
B[a, u,-2,0,0,-2] & =B[a, u, 0,-2,-2,0] \\
B[a, u,-1,0,0,-1] & =B[a, u, 0,-1,-1,0]
\end{aligned}
$$

Taking symmetry eq. (9b) of ref. [16] into account

$$
B[a, u, \kappa, \eta, \mu, \nu]=B[-a, u,-\kappa,-\eta,-\mu,-\nu]
$$

Notice the difference with respect to eq. (9.b) of ref. [16], where an additional factor $2 \rho$ is present in the r.h.s., $-a \rightarrow-a-2 \rho$.

And also the symmetry eq. (9c)

$$
B[a, u, \kappa, \eta, \mu, \nu]=-(-1)^{\kappa-\nu} \sqrt{\frac{G\left[a+e_{\kappa}\right] G\left[a+e_{\mu}\right]}{G[a] G\left[a+e_{\kappa}+e_{\eta}\right]}} B\left[a+e_{\mu},-6-u,-\mu, \kappa, \nu,-\eta\right]
$$


Open Access. This article is distributed under the terms of the Creative Commons Attribution License (CC-BY 4.0), which permits any use, distribution and reproduction in any medium, provided the original author(s) and source are credited.

\section{References}

[1] R.J. Baxter, Exactly solved models in statistical mechanics, Academic Press, London, U.K. (1982).

[2] V. Belavin and D. Gepner, Three blocks solvable lattice models and Birman-Murakami-Wenzl algebra, Nucl. Phys. B 938 (2019) 223 [arXiv:1807.05603] [INSPIRE].

[3] V. Belavin and D. Gepner, On the algebraic approach to solvable lattice models, JHEP 02 (2019) 033 [arXiv: 1810.07959] [INSPIRE].

[4] D. Gepner, Foundations of rational quantum field theory, I, CALT-68-1825, (1992) [hep-th/9211100] [INSPIRE].

[5] H.N.V. Temperley and E.H. Lieb, Relations between the 'percolation' and 'colouring' problem and other graph-theoretical problems associated with regular planar lattices: some exact results for the 'percolation' problem, Proc. Roy. Soc. Lond. A 322 (1971) 251 [inSPIRE].

[6] J.S. Birman and H. Wenzl, Braids, link polynomials and a new algebra, Trans. Amer. Math. Soc. 313 (1989) 249.

[7] J. Murakami, The Kauffman polynomial of links and representation theory, Osaka. J. Math. 24 (1987) 745.

[8] G. Kuperberg, The quantum $G_{2}$ link invariant, Int. J. Math. 05 (1994) 61.

[9] E. Kalfagianni, On the $G_{2}$ link invariant, J. Knot Theor. Ramificat. 02 (1993) 431.

[10] Yu. G. Stroganov, A new calculation method for partition functions in some lattice models, Phys. Lett. A 74 (1979) 116 [inSPIRE].

[11] R.J. Baxter, The inversion relation method for some two-dimensional exactly solved models in lattice statistics, J. Statist. Phys. 28 (1982) 1 [INSPIRE].

[12] V.F.R. Jones, Notes on subfactors and statistical mechanics, Int. J. Mod. Phys. A 5 (1990) 441 [INSPIRE].

[13] M. Wadati, T. Deguchi and Y. Akutsu, Exactly solvable models and knot theory, Phys. Rept. 180 (1989) 247 [inSPIRE].

[14] N. Yu. Reshetikhin and V.G. Turaev, Ribbon graphs and their invariants derived from quantum groups, Commun. Math. Phys. 127 (1990) 1 [INSPIRE].

[15] G.W. Moore and N. Seiberg, Polynomial equations for rational conformal field theories, Phys. Lett. B 212 (1988) 451 [inSPIRE].

[16] A. Kuniba and J. Suzuki, Exactly solvable $G_{2}^{(1)}$ solid-on-solid models, Phys. Lett. A 160 (1991) 216. 